\title{
General Theory and Practice of Basic Models in the Building of Hydroacoustical Antennas
}

\author{
Zvonimir Milošić \\ Institute for Research and Development of Defence Systems - MoD, HR 10000 Zagreb, \\ Croatia
}

\section{Introduction}

The general form of a model of electromechanical vibration system with concentrated parameters, presented in Fig. 1.1., is a good example for the input in the analysis of transmit and receive sensitivity of hydroacoustical transducer. A well known model of electromechanical elements transformed in electrical substitute components of hydroacoustic transducer, presents a widely known procedure for de-embedding its different characteristics which are necessary in producing of antenna. This procedure has been derived on an idealized model of block scheme of hydroacoustic transducer on the level of electrical analogy of all electric and mechanical parameters. In a hydroacoustical system with one piezoelectric element (hydrophone), different values of a phase angle at electric clamps come from phase relations of mechanical force and vibration velocity in a mechanical vibration system.
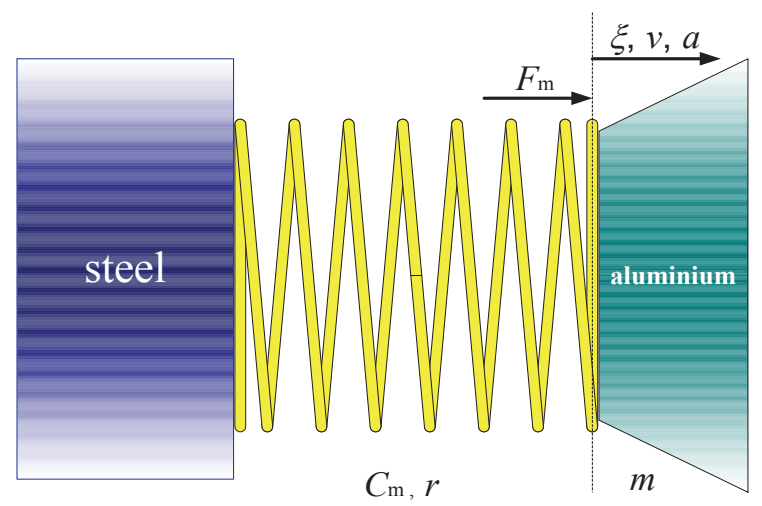

Fig. 1.1. Model of illustration of general mechanical system with potential mechanical force, vibrating spring and concentrated parameters of mass

This general form of a mechanical model of vibration of medium with concentrated mechanical parameters presented as a starting point of analysis of receive sensitivity of hydroacoustical transducers on the basis of well known differential equations (Aronov \& 
Matveev 1953) and with Laplace transformation has very complex mathematical solves which in the domain of the complex variable have a special usability and meaning.

On the level of mathematical solves in the domain of the complex variable harmonic motions are characterised by the values as follows,

$F(\mathrm{~N})=F_{\mathrm{m}}(\mathrm{N})$-the amplitude of mechanical force on the elastic system of motion and vibration is at the same time an active generator of mechanically forced vibrations/oscillations. The mechanical force brings piezoelectric system in interaction with piezoelectric effect of electrical force with mechanical mass of the system. In that case, $F(t)$ as mechanical force in dependence of time $t$, is given in a general form as follows

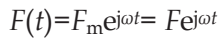

The amplitude of harmonic motion, elongation $-\xi_{\mathrm{m}}(\mathrm{m})$, or the highest displacement of material point of elastic medium to the central position of motion, and $\xi(t)$ as displacement of material point of elastic medium to the point at the beginning as a function of time $t$ in general form, can be in the phase of moving to the mechanical force and it can be written in mathematical form as follows

$$
\xi(t)=\xi_{\mathrm{m}} \mathrm{e}^{\mathrm{i}(\omega t-\psi)}
$$

where is

$T(\mathrm{~s})$-time as a period of one vibration of material point of mass,

$\omega(\mathrm{rad} / \mathrm{s})$-angular velocity as frequency of mechanical oscillator or number of vibrations in $2 \pi$ seconds,

$t(\mathrm{~s})$-time,

$\psi(\mathrm{rad})$-phase angle of phase shift of deviation of material point in given time $t$ in the reference to mechanical force $F$ damped with mass $m$

$v(\mathrm{~m} / \mathrm{s})$-velocity as value of velocity of projection of material point on axes which is parallel to elongation, this is explained as the change of distance in time

$$
v(t)=\frac{\partial \xi}{\partial t}
$$

or simpler in a symbolic form, when we have

$$
v=v(t)=\xi^{\prime}
$$

As the first derivation of moving in time, the velocity of material point in elastic medium is

$$
\begin{aligned}
& v=\mathrm{j} \omega \cdot \xi_{\mathrm{m}} \cdot \mathrm{e}^{\mathrm{j}(\omega t-\psi} \\
& v=v_{\mathrm{m}} \cdot \mathrm{e}^{\mathrm{j}(\omega t-\psi+\Pi / 2)}
\end{aligned}
$$

with amplitude of this velocity $v_{\mathrm{m}}=\omega \cdot \xi_{\mathrm{m}}$.

Furthermore, the acceleration of a particle in harmonic motion $a(t)$ in a mathematical form is equal

$$
a(t)=\frac{\partial v}{\partial t}=\frac{\partial^{2} \xi}{\partial t^{2}}
$$

or simpler, in a symbolic form we can write 


$$
a=\xi^{\prime \prime}
$$

After a double derivation of displacement $\xi(t)$ as a variable in time $t$ we have a symbolic form as follows

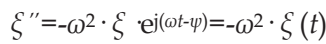

Using the known meaning of parameters in mechanics, we can arrive to the known differential equation as follows

$\mathrm{C}(\mathrm{m} / \mathrm{N})$-constant of mechanical elasticity of the vibration system of concentrated parameters,

$m(\mathrm{~kg})$-mass of material point,

$r(\mathrm{Ns} / \mathrm{m})$-resistance of friction and mechanical losses in the vibration system

$\delta(1 / \mathrm{s})$-factor of suppressing of mechanical vibration defined by $r$ and $m$ in mathematical relation as follows

$$
\delta=\frac{r}{2 m}
$$

By introducing partial expressions for all forms of energies contained in different forces, we have the force of elastic system $F_{\mathrm{e}}$ as follows

$$
F_{\mathrm{e}}=\frac{\xi(t)}{C_{\mathrm{m}}}=\frac{\xi}{C_{\mathrm{m}}}
$$

The force of acceleration $F_{\mathrm{a}}$ of mass $m$ in moving is

$$
F_{\mathrm{a}}=m \cdot a(t)=m \cdot \xi^{\prime \prime}
$$

The force of resistance of friction and mechanical losses in the vibration system is

$$
F_{\mathrm{t}}=r v(t)=r \cdot \xi^{\prime}
$$

Module of elongation $\xi$ as function of vibration frequency of mass

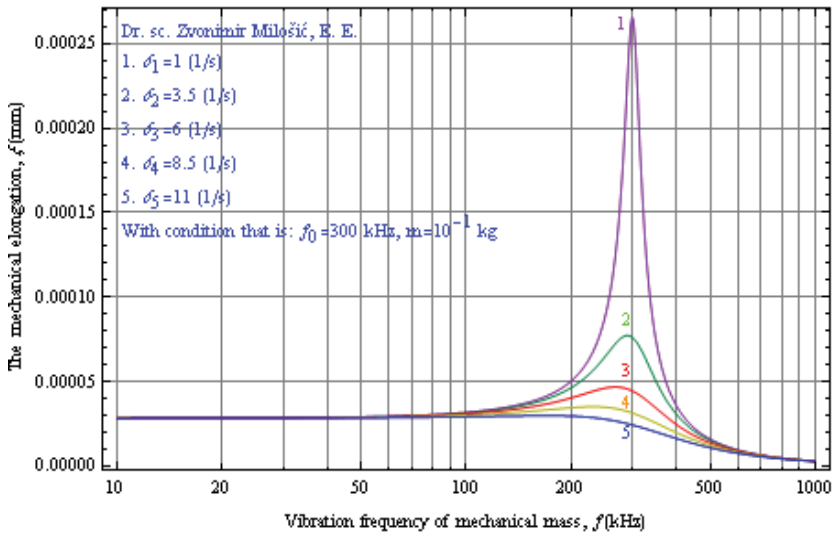

Fig. 1.2 Dependence of module of elongation of mass $\xi(f)$ with concentrated parameters on working frequency $f$ 
The balance of energy is made by the balance of all forces in a harmonic vibration system and then we get a differential equation as follows

$$
m \cdot \xi^{\prime \prime}+r \cdot \xi^{\prime}+\xi / C_{\mathrm{m}}=F \cdot \mathrm{e}^{\mathrm{j} \omega t}
$$

By using mathematical expression for $\omega_{0}$ as a parameter in conditions of mechanical resonance, we have

$$
\omega_{0}^{2}=\frac{1}{m \cdot C_{m}}
$$

and with a factor of choking and damping of mechanical vibration $\delta$ in (1.9) we have a symbolic form of differential equation as follows

$$
\xi^{\prime \prime}+2 \delta \cdot \xi^{\prime}+\omega_{0}^{2} \cdot \xi=\frac{F}{m} \cdot \mathrm{e}^{j \omega t}
$$

To solve this differential equation we need to determine the beginning conditions of displacement of a material point of the vibration system in $t=0$. By using differential solves of stationer state we can get Fig. 1.2 as a model of physical state of the matter. We have two important fields in the frequency domain of hydroacoustic transducer sensitivity: constant value of displacement of material point in medium and the field of resonance.

Dependence of phase angle of impedance $\omega_{z}$ of vibrational frekvency of mecharical mass

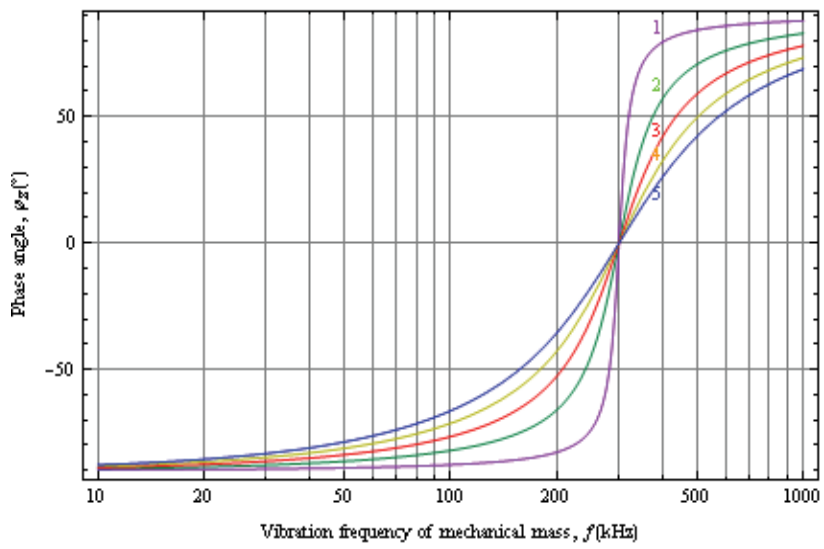

Fig. 1.3 Dependence of phase angle of working frequency $f$ of elongation of mass $\xi(f)$ with concentrated parameters as function on vibration frequency

On the other hand, according to Fig. 1.3, the values of phase angles with too high dispersion of different parameters at a receive multi-element antenna can have negative influences on the parameters which are very important for detection, in the resolution and in the less of great ranges of sonars and medicine ultrasound scanners, too. Accordingly, with the analysis of the behaviour of mathematical functions of sensitivity and sources of wide dispersion of phase angles of antenna elements at hydroacoustical and ultrasound transducers, we can choose the best model for an optimal range in the given system. 


\section{The accordance of a model of mechanical vibration system with concentrated parameters and composed hydroacoustical transducer}

On the basis of their mathematical solutions we analyze the frequency characteristic of receive sensitivity of hydrophone. In a hydroacoustical antenna system with one piezoelectric element (hydrophone) the values of phase angles on electrical clamps come from phase relations of mechanical vibrations in an electromechanical vibration system of single elements.

The value of a phase angle is not of great importance and influence on other parameters of single elements. On the other hand, the values of a phase angle with too wide dispersion of different parameters of single element at receive multi-elemental antenna, can have negative influence on parameters, which is very important for great ranges of sonars and medicine ultrasound scanners, too. Accordingly, with the analysis of the behavior of mathematical functions of sensitivity and sources of wide dispersion of phase angles of antenna elements at hydroacoustical and ultrasound transducers, we can choose the best model for optimal range in the given system.

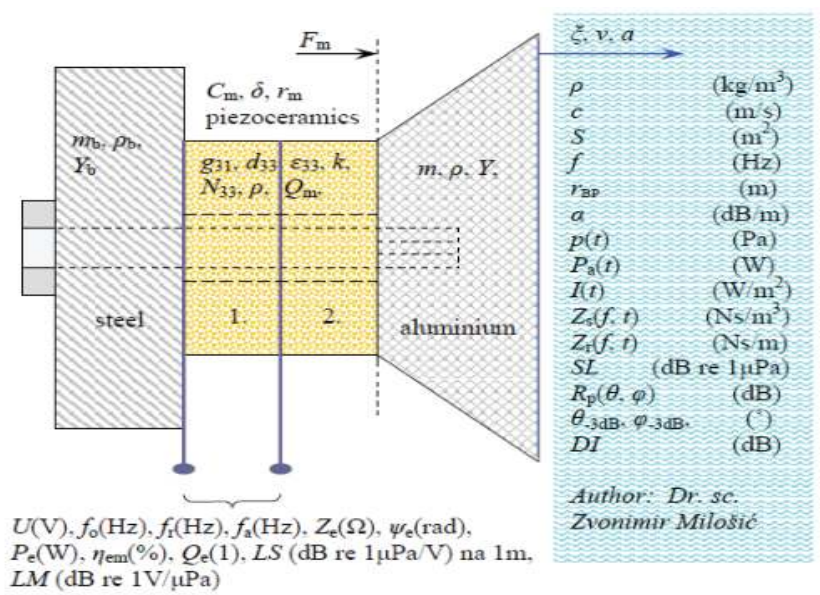

Fig.2.1 Model of hydroacoustical composed transducer with general positions of all parameters, ones on the side of water and others on the side of electrical ends and inside their mechanical body, (Milošić 2004)

\subsection{Theory of hydroacoustical composed transducer in the form of two-port network(6pt)}

In this chapter is presented the procedure of calculating some characteristics of hydroacoustical antenna. The procedure is derived on an idealized model of a electromechanical block scheme of hydroacoustical transducer, (Masson 1950, Beranek 1954, Kinsler\&Frey 1962, Caruthers 1977, Sverdlin 1976, 1980, Bogorodskii\&All 1983, Wilson 1998).

We analyze the complex behavior of mechanical vibration system of hydroacoustic transducer analyzing at electrical analogy of electric impedance on its electrical ends in 
specific conditions. We more often use the block scheme of hydroacoustical transducer in Fig. 2.1.1 as electromechanical two-port network devices in theory and practice of the system than in the construction analyses of the transducer.

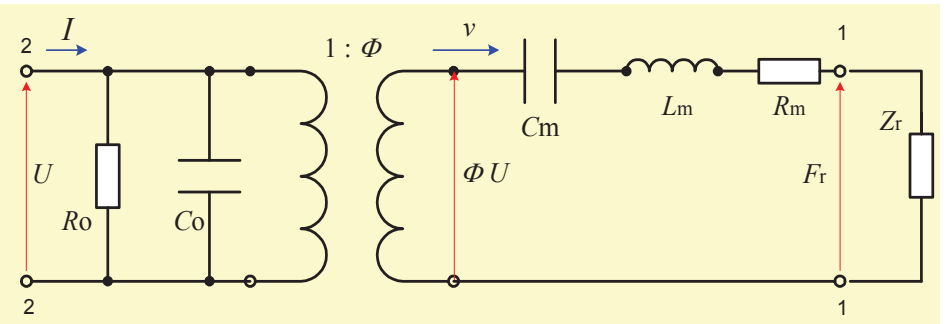

Fig. 2.1.1 Model of electromechanical block scheme of hydroacoustical composed transducer in the form of two-port network devices for determining electrical frequency characteristic of sensitivity

Mutual dependence of mechanical and electrical components of parameters is defined by coefficient of mechanical transformation $\Phi(\mathrm{N} / \mathrm{V})$. The balance of electromechanical vibration system must be realized at all lines of spreading of the sound, from source of electric energy to impedance of radiation of acoustical energy and reversely.

By using the model of electromechanical block scheme Fig. 2.1.1 of hydroacoustical transducer on the left electrical circuit, we can write that is

$$
I=U Y_{0}+\Phi v
$$

Mechanical vibration system in the second circuit on the right is in balance with equation (2.1.2) also, and then yield

$$
F=\Phi U+v Z_{\mathrm{m}}
$$

But, on the other hand, we can introduce mechanical force $F$ and vibration of velocity of the transducer face of effective surface in equation (2.1.3), as

$$
v=-\frac{F}{Z_{r}}
$$

In this case, we have three equations as starting points for different directions with solves of different important parameters of hydroacoustic transducer.

\subsection{The de-embedding of electric input impedance of hydroacoustical transducers}

By using three equations (2.1.1), (2.1.2) and (2.1.3) we can get electric input impedance at port 2-2 in general form as in equation (2.2.1) which is in accordance with equation (2.14) at reference (Caruthers 1977). Electric form of block scheme is given in Fig. 2.2.1.

$$
Z_{\text {in }}=R_{\text {in }}+\mathrm{j} x_{\text {in }}
$$

In accordance with equations above, we have a general form of real and imaginary part of impedance, then yield 


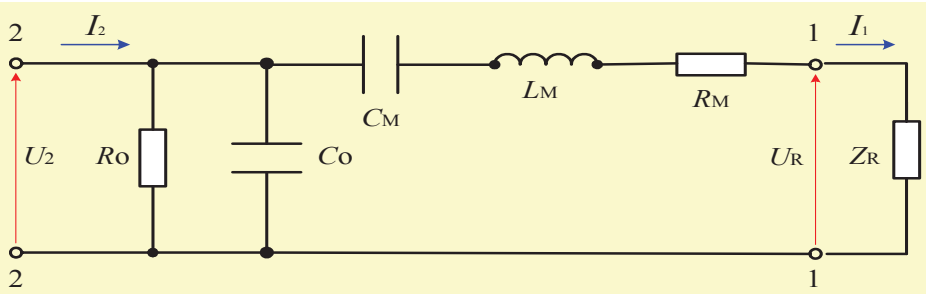

Fig. 2.2.1 Electric block scheme is given in a general form of equation of electric parameters on the input and electric analogy of mechanical parameters of vibration system

$$
\begin{aligned}
& R_{\text {in }}=R_{\mathrm{t}}=\frac{R_{0} x_{0}\left(R_{\mathrm{MR}}{ }^{2}+x_{\mathrm{M}}{ }^{2}\right)\left(R_{0} R_{\mathrm{MR}} x_{0}+R_{\mathrm{MR}}{ }^{2} x_{0}+x_{0} x_{\mathrm{M}}{ }^{2}\right)}{\left(R_{0} R_{\mathrm{MR}} x_{0}+R_{\mathrm{MR}}{ }^{2} x_{0}+x_{0} x_{\mathrm{M}}^{2}\right)^{2}+\left(R_{0} x_{0} x_{M}+R_{0} R_{\mathrm{MR}}{ }^{2}+R_{0} x_{M}\right)^{2}} \\
& x_{\text {in }}=x_{\mathrm{t}}=\frac{R_{0} x_{0}\left(R_{\mathrm{MR}}{ }^{2}+x_{\mathrm{M}}{ }^{2}\right)\left(R_{0} x_{\mathrm{M}} x_{0}+R_{\mathrm{MR}}{ }^{2} R_{0}+R_{0} x_{\mathrm{M}}{ }^{2}\right)}{\left(R_{0} R_{\mathrm{MR}} x_{0}+R_{\mathrm{MR}}{ }^{2} x_{0}+x_{0} x_{\mathrm{M}}{ }^{2}\right)^{2}+\left(R_{0} x_{0} x_{M}+R_{0} R_{\mathrm{MR}}{ }^{2}+R_{0} x_{M}{ }^{2}\right)^{2}}
\end{aligned}
$$

where is

$\Phi(\mathrm{N} / \mathrm{V})$-factor of electro-mechanical transformation of electrical parameters in associated mechanic parameters and reverse (electric voltage $U(\mathrm{~V})$ in mechanical force $F(\mathrm{~N})$ ),

$R_{0}(\Omega)$-resistor of electrical losses in piezoelectric material, can be measured with electrical tools at input electric clamps of transducer,

$C_{0}(\mathrm{~F})$-electrical capacity of condenser of piezoelectric material at input clamp of electromechanical transducer, can be calculated from catalogue data and mechanical dimensions or measured with electrical tools at input electric clamps of transducer,

$Z_{0}(\Omega)$-the clamped electric impedance as reciprocity value of electric admittance $Y_{0}$ calculated from data of parallel connection of $R_{0}$ and $C_{0}$ to Fig. 2.2.1,

$\mathrm{C}_{\mathrm{M}}(\mathrm{F})$-electrical capacity as analogous of mechanical system as mechanical capacitance defined as $C_{\mathrm{m}}$. It can be calculated from catalogue data and data of special measured tools of transducer,

$$
\mathrm{C}_{\mathrm{M}}=\Phi^{2} \mathrm{C}_{\mathrm{m}}
$$

$L_{\mathrm{M}}(\mathrm{H})$-electric inductivity as analogous of elasticity of mechanical system defined as $L_{\mathrm{m}}$. It can be calculated from catalogue data and data of measured tools of transducer,

$$
L_{\mathrm{M}}=L_{\mathrm{m}} / \Phi^{2}
$$

$R_{\mathrm{M}}(\Omega)$-electric resistor as analogous of mechanical resistance of mechanical system defined as $R_{\mathrm{m}}$. It can be calculated from catalogue data and data of measured tools of transducer,

$$
R_{\mathrm{M}}=R_{\mathrm{m}} / \Phi^{2}
$$

$Z_{\mathrm{M}}(\Omega)$-electric impedance as analogous of mechanical impedance of mechanical system defined as $Z_{\mathrm{m}}$. We calculate it from calculated and measured data as follows,

$$
Z_{\mathrm{M}}=R_{\mathrm{M}}+\mathrm{j} x_{\mathrm{M}}=R_{\mathrm{M}}+\mathrm{j}\left(x_{\mathrm{L}}+x_{\mathrm{C}}\right)=R_{\mathrm{M}}+\mathrm{j}\left(\omega L_{\mathrm{M}}-1 / \omega \mathrm{C}_{\mathrm{M}}\right)=Z_{\mathrm{m}} / \Phi^{2}
$$

$R_{R}(\Omega)$-electric resistor as analogous of acoustical resistance of acoustical effective radiated surface of system defined as $R_{\mathrm{r}}$ which presents the resistance of radiation of value of sound energy, can be calculated from catalogue data and data of measured tools of transducer, 


$$
R_{\mathrm{R}}=R_{\mathrm{r}} / \Phi^{2}=\rho c A K_{R} / \Phi^{2}
$$

$x_{\mathrm{R}}(\Omega)$-electric reactive resistor as analogous of acoustical reactance of acoustical effective radiated surface of system defined as $x_{\mathrm{r}}$ which presents the reactance of radiation system of sound energy, can be calculated from catalogue data and data of measured tools of transducer. In fact we make it to be zero or to obtain to zero $\left(x_{R} \rightarrow 0\right)$,

$$
x_{\mathrm{R}}=x_{\mathrm{r}} / \Phi^{2}=\rho c A K_{x} / \Phi^{2}
$$

$A\left(\mathrm{~m}^{2}\right)$-effective aperture, effective surface of radiated face of transducer,

$\rho\left(\mathrm{kg} / \mathrm{m}^{3}\right)$-specific mass of water as medium of spreading of sound,

$c(\mathrm{~m} / \mathrm{s})$-velocity of sound in water or people's body. Velocity in the sea is complex function of a lot of parameters, but approximately, we can take that $c \approx 1500 \mathrm{~m} / \mathrm{s}$

$Z_{R}(\Omega)$-electrical value of acoustic radiation impedance $Z_{r}$ presents the value and character of radiated acoustic energy in space. We calculate it from data of $R_{\mathrm{R}}$ and $x_{\mathrm{R}}$ as follows

$$
Z_{\mathrm{R}}=R_{\mathrm{R}}+\mathrm{j} x_{\mathrm{R}}=\mathrm{Z}_{\mathrm{r}} / \Phi^{2}=\rho c A\left(K_{\mathrm{R}}+K_{x}\right) / \Phi^{2}
$$

$K_{R}(1)$-coefficient of acoustic resistance $R_{\mathrm{r}}$ as real part of acoustical radiated impedance. By using Bessel and Struve special functions we can calculate it at piston model of transducer (Caruthers 1977) and it is shown in Fig. 2.2.2, but mainly using cylindrical model of transducer, we can calculate it with equation by using special Hankel functions (Rževkin 1960, Smarišev 1973) or we take data in Fig. 2.2.3.

$K_{x}(1)$-coefficient of acoustic reactance $x_{\mathrm{r}}$ as imaginary part of acoustically radiated impedance at mentioned figures,

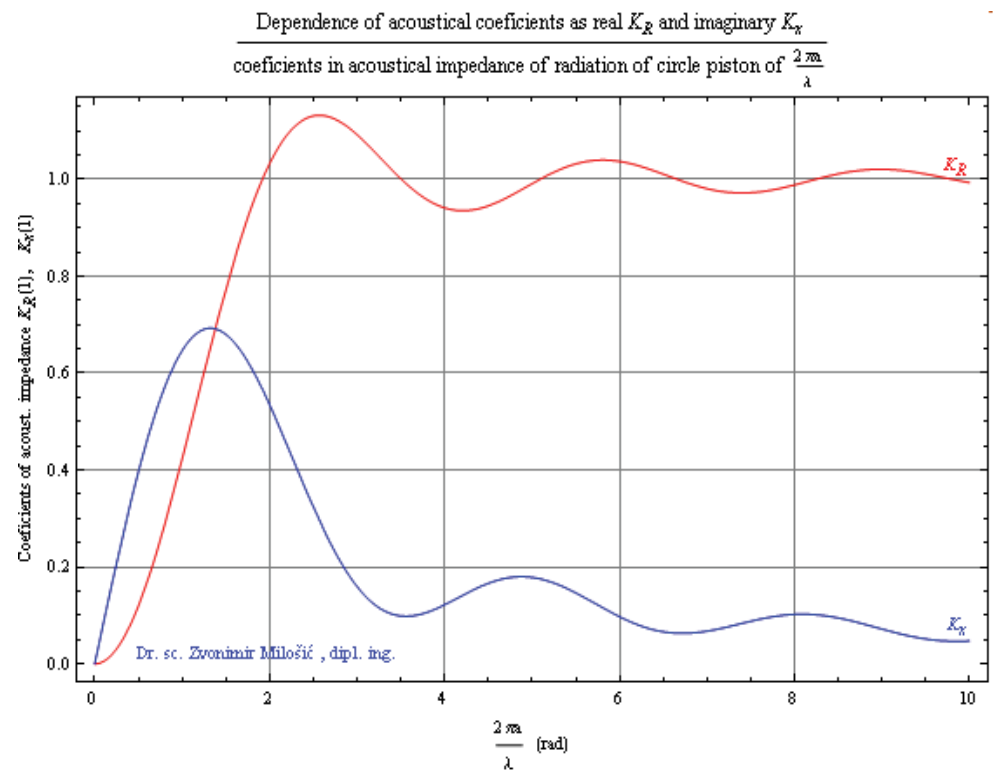

Fig. 2.2.2 Graphic presentation of data for $K_{R}$ and $K_{x}$ at circle piston model of transducer 


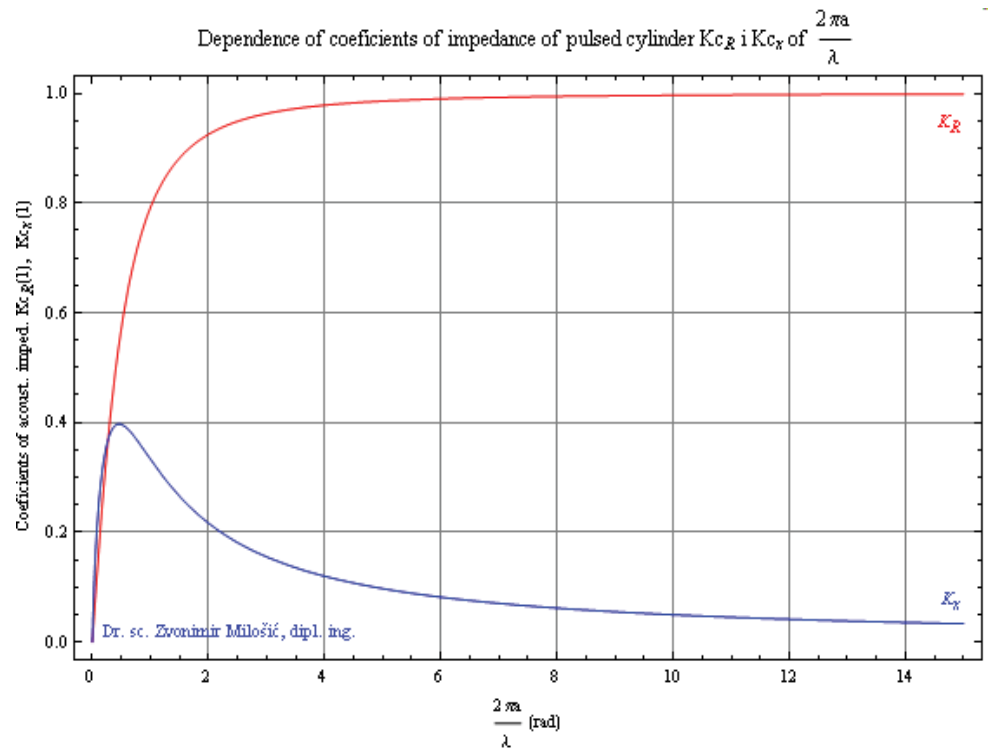

Fig. 2.2.3 Graphic presentation of data for $K_{R}$ and $K_{x}$ at cylindrical model of transducer

In fact, according to the graph in Fig. 2.2.3, we can choose a transducer with cylindrical radiated face as a great advantage because in an equal volume cylinder, in relation to the piston transducer or to the composed transducer, it has a higher voltage sensitivity and lower reactive radiated impedance. Keeping in line with this logic, using transducers with cylindrical radiated face at circle (barrel) antennas systems in receive is very often.

If we assume that $R_{0}$ is infinite or increase to infinite, we have good conditions for special impedance analysis in frequency domain. By using mathematical operator 'limes' at expressions (2.2.2) and (2.2.3) with $R_{0} \rightarrow 0$ we have shorter equations as follows

$$
\begin{gathered}
R_{\mathrm{in}}=R_{\mathrm{t}}=\frac{R_{\mathrm{MR}} x_{0}{ }^{2}\left(R_{\mathrm{MR}}{ }^{2}+x_{\mathrm{M}}{ }^{2}\right)}{R_{\mathrm{MR}}{ }^{2} x_{0}{ }^{2}+\left(R_{\mathrm{MR}}{ }^{2}+x_{0} x_{M}+x_{M}{ }^{2}\right)^{2}} \\
x_{\text {in }}=x_{\mathrm{t}}=\frac{x_{0}\left(R_{\mathrm{MR}}{ }^{2}+x_{\mathrm{M}}{ }^{2}\right)\left(R_{\mathrm{MR}}{ }^{2}+x_{\mathrm{M}} x_{0}+x_{\mathrm{M}}{ }^{2}\right)}{R_{\mathrm{MR}}{ }^{2} x_{0}{ }^{2}+\left({R_{\mathrm{MR}}}^{2}+x_{0} x_{M}+x_{M}\right)^{2}}
\end{gathered}
$$

By using different conditions of maximal and of minimal value of module of electric impedance $Z_{\mathrm{t}}$ and admittance $Y_{\mathrm{t}}$ and on condition that $x_{\mathrm{t}}=x_{\mathrm{in}}=0$ if it is possible, we can deembed the important series of frequencies as follows: $f_{\mathrm{m}}<f_{\mathrm{s}}<f_{\mathrm{r}}<f_{\mathrm{a}}<f_{\mathrm{p}}<f_{\mathrm{n}}$. Four of them: serial frequency $f_{\mathrm{s}}$, resonance frequency $f_{\mathrm{r}}$, anti-resonance frequency $f_{\mathrm{a}}$ and frequency of parallel resonance of system $f_{\mathrm{p}}$ in given conditions can be calculated to expressions as follows

$$
f_{\mathrm{s}}=\lim _{R_{\mathrm{MR}} \rightarrow 0} f_{\mathrm{r}}=\frac{1}{2 \pi \sqrt{L_{\mathrm{M}} C_{\mathrm{M}}}}
$$



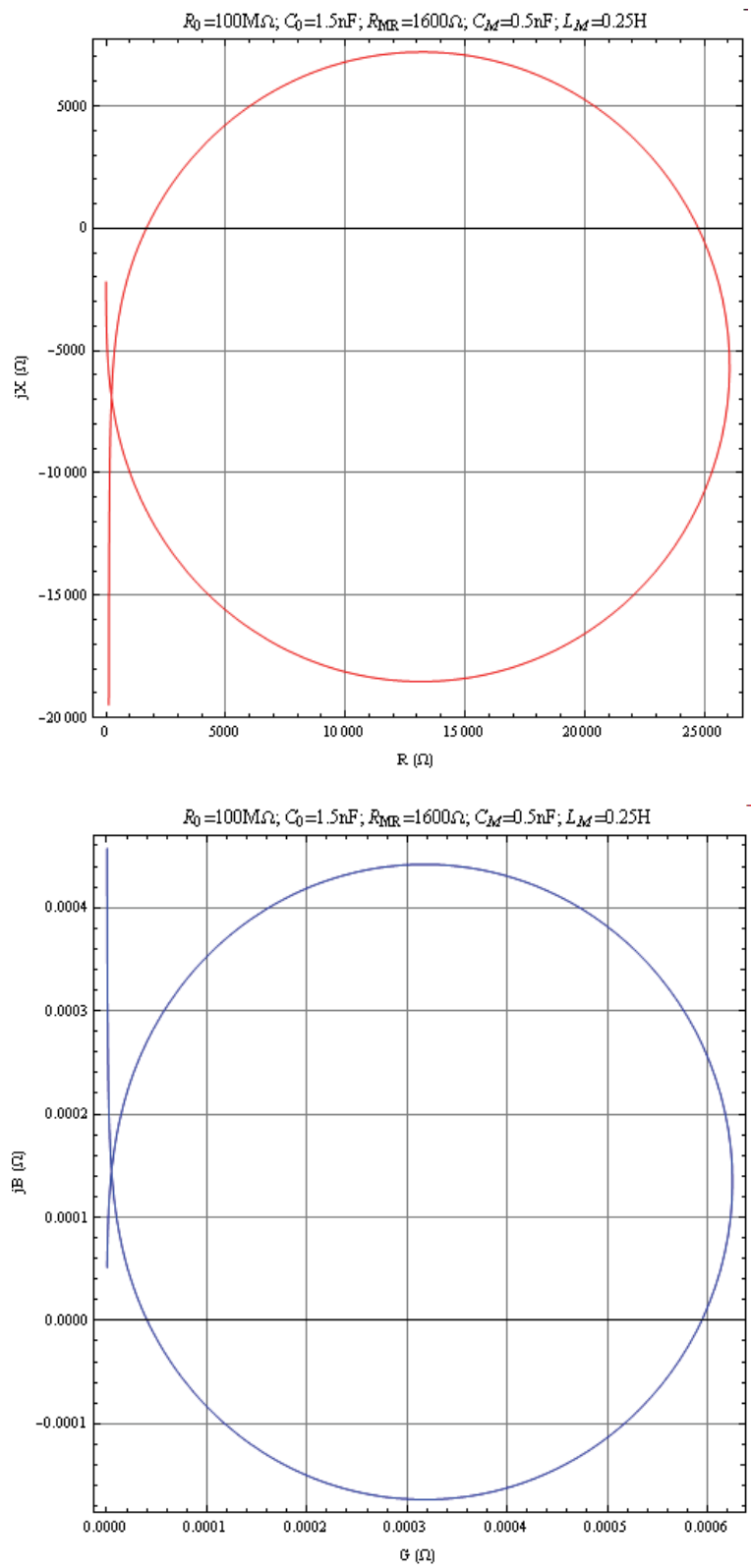

Fig. 2.2.4 Circle diagrams of input electric impedance and admittance of piezoelectric transducer at $R_{\mathrm{MR}}=1600 \Omega, L_{\mathrm{M}}=0.25 \mathrm{H}, C_{\mathrm{M}}=0.5 \mathrm{nF}$ i $C_{0}=1.5 \mathrm{nF}, R_{\mathrm{c}}=50 \Omega$ 


$$
\begin{aligned}
& f_{\mathrm{r}}=\frac{1}{2 \pi} \cdot \sqrt{\frac{1}{2 L_{\mathrm{M}} C_{0}}+\frac{1}{L_{\mathrm{M}} C_{\mathrm{M}}}-\frac{R_{\mathrm{MR}}{ }^{2}}{2 L_{\mathrm{M}}{ }^{2}}-\sqrt{\frac{R_{\mathrm{MR}}{ }^{4}}{4 L_{\mathrm{M}}{ }^{4}}+\frac{1}{4 L_{\mathrm{M}}{ }^{2} C_{0}{ }^{2}}-\frac{R_{\mathrm{MR}}{ }^{2}}{L_{\mathrm{M}}{ }^{3} C_{\mathrm{M}}}-\frac{R_{\mathrm{MR}}{ }^{2}}{2 L_{\mathrm{M}}{ }^{3} C_{0}}}}(\mathrm{~Hz}) \\
& f_{\mathrm{a}}=\frac{1}{2 \Pi} \cdot \sqrt{\frac{1}{2 L_{\mathrm{M}} C_{0}}+\frac{1}{L_{\mathrm{M}} C_{\mathrm{M}}}-\frac{R_{\mathrm{MR}}{ }^{2}}{2 L_{\mathrm{M}}{ }^{2}}+\sqrt{\frac{R_{\mathrm{MR}}{ }^{4}}{4 L_{\mathrm{M}}{ }^{4}}+\frac{1}{4 L_{\mathrm{M}}{ }^{2} C_{0}{ }^{2}}-\frac{R_{\mathrm{MR}}{ }^{2}}{L_{\mathrm{M}}{ }^{3} C_{\mathrm{M}}}-\frac{R_{\mathrm{MR}}{ }^{2}}{2 L_{\mathrm{M}}{ }^{3} C_{0}}}} \\
& f_{\mathrm{p}}=\lim _{R_{\mathrm{MR}} \rightarrow 0} f_{\mathrm{a}}=\frac{1}{2 \pi} \sqrt{\frac{C_{\mathrm{M}}+C_{0}}{L_{\mathrm{M}} C_{\mathrm{M}} C_{0}}}
\end{aligned}
$$

We de-embed two of the frequencies: $f_{\mathrm{m}}$ in relation to the minimum of module of electric impedance $Z_{\mathrm{t}}$ and $f_{\mathrm{n}}$ as frequency to the minimal value of admittance $Y_{\mathrm{t}}$ at circle impedance and admittance diagrams respectively. Yield at example, Fig. 2.2.4.

\subsection{The matching of electric impedance of hydroacoustical transducers}

In accordance with maximal efficiency and maximal transmission of electric energy and maximal relations of signal/noise, we can calculate parameters of matching with electric transformer to Fig. 2.3.1 or with LC transformer to Fig. 2.3 .3 (Milošić 1978, 2004, 2010). On the other hand, in accordance with maximal efficiency for noise factor we need to make the matching of electric impedance in conditions for the lowest noise (Jelaković 1973).

The most suitable using of electric transformer is in active systems of transmitter with narrow band pass and stable transmitting frequency.

General forms of original equations for matching in the system with electric transformers are taken over from the mentioned literature (Milošić 2010) and we have as follows

$$
w_{\mathrm{s}}=\sqrt{\frac{\left|Z_{\mathrm{tc}}\right|}{\omega A_{L} \cdot\left|\sin \psi_{\mathrm{tc}}\right|}}=\sqrt{\frac{\left|Z_{\mathrm{tc}}\right|}{2 \Pi f A_{L}} \cdot \frac{\sqrt{1+\tan ^{2} \psi_{\mathrm{tc}}}}{\left|\tan \psi_{\mathrm{tc}}\right|}}
$$

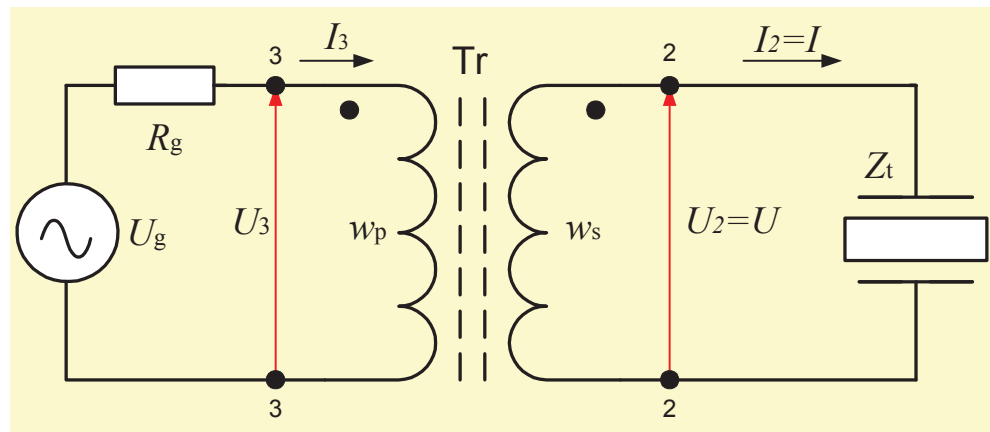

Fig. 2.3.1 Electric block scheme is given for general form of equation of electric parameters of electric transformer on input impedance of transducer $Z_{t}$ (Milošić 1976, 2004, 2010) 


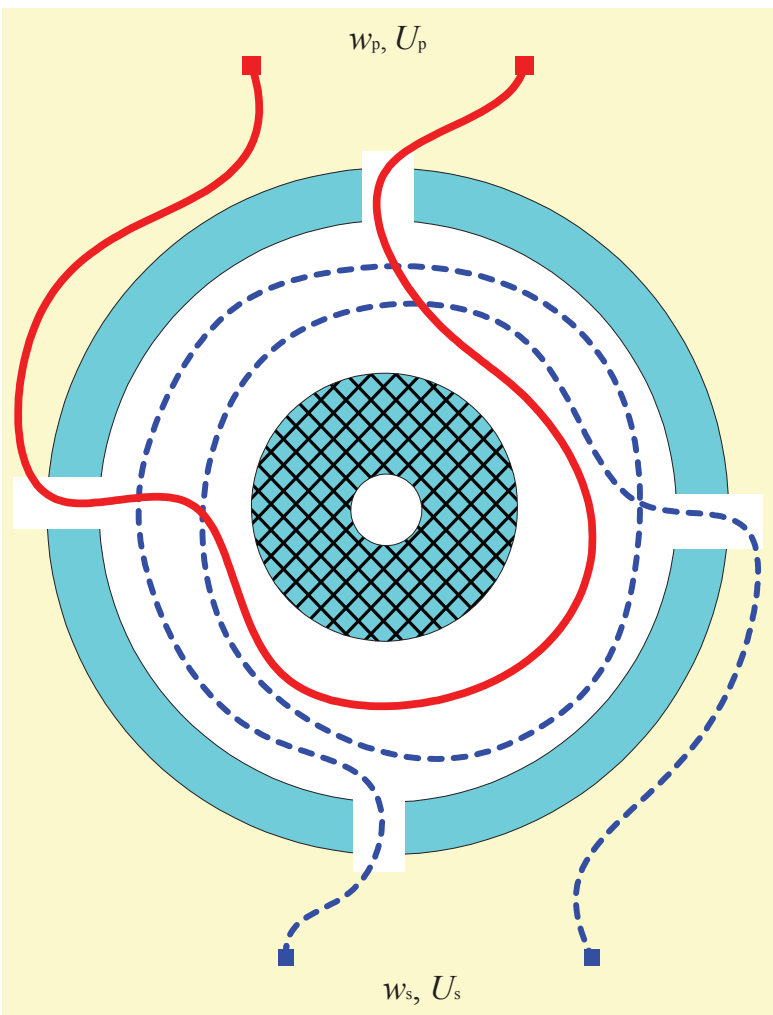

Fig. 2.3.2 The illustration of the turn spacing with the coiling conduct and the decimal value of the turn on the four-slot ferrite core, $w_{\mathrm{p}}=0.75$ turn and $w_{\mathrm{s}}=1.75$ of the turns in theory and in practice (Milošić 1978, 2010)

$$
\begin{gathered}
w_{\mathrm{p}}=\sqrt{\frac{R_{0}}{A_{L} \omega \cdot\left|\tan \psi_{\mathrm{tc}}\right|}}=\sqrt{\frac{R_{0}}{2 \pi f A_{L} \cdot\left|\tan \psi_{\mathrm{tc}}\right|}} \\
\left(\frac{w_{s}}{w_{p}}\right)^{2}=\frac{R_{\mathrm{t}}}{R_{0}} \cdot \frac{1}{\cos ^{2} \psi_{\mathrm{t}}}
\end{gathered}
$$

On the other hand, $L C$ electric transformer is good in wide band sonar systems in receiving of signals and at physically very composed systems of antenna. Given parameters of LC transformers can be calculated very fast and precisely to (2.3.4), (2.3.5) and (2.3.6).

$$
C_{\mathrm{tr}}=\frac{R_{\mathrm{t}}-R_{0}}{2 \pi f \cdot\left|Z_{\mathrm{t}}\right| \cdot\left(-R_{0} \sin \psi_{\mathrm{t}}+\sqrt{R_{0} R_{\mathrm{t}}-R_{0}^{2} \cos ^{2} \psi_{\mathrm{t}}}\right)}
$$




$$
\begin{aligned}
& L_{\mathrm{tr}}=\frac{1}{2 \pi f} \cdot \frac{\left|Z_{\mathrm{t}}\right|^{2} x_{C}-x_{C}{ }^{2}\left|Z_{\mathrm{t}}\right| \cdot \sin \psi_{\mathrm{t}}}{\left|Z_{\mathrm{t}}\right|^{2}+x_{C}{ }^{2}-2 x_{C}\left|Z_{\mathrm{t}}\right| \cdot \sin \psi_{\mathrm{t}}} \\
& \tan \psi_{\text {in }}=\tan \psi_{\mathrm{t}}+\frac{R_{\mathrm{t}}}{2 \pi f A_{L} w_{\mathrm{s}}{ }^{2}} \cdot \frac{1}{\cos ^{2} \psi_{\mathrm{t}}}
\end{aligned}
$$

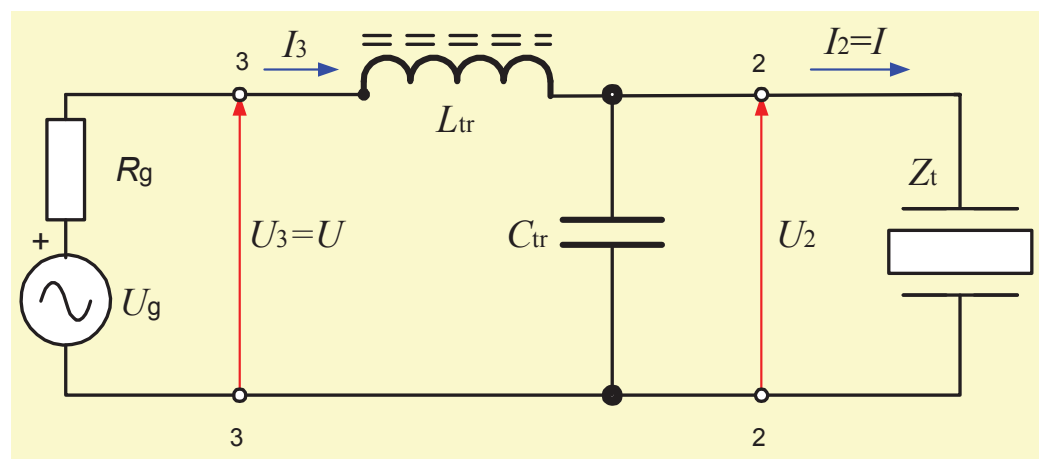

Fig. 2.3.3 Electric block scheme is given for general form of equation of electric parameters of $L C$ transformer on input impedance of transducer $Z_{t}$ to reference (Milošić 1978, 2010)

\section{The de-embedding of the level of transmitting voltage sensitivity of transducers $-L S(\mathrm{~dB}$ re $1 \mu \mathrm{Pa} / \mathrm{V}$ at $1 \mathrm{~m})$}

Keeping in line with the de-embedding of the level of transmitting voltage sensitivity of transducer we need to eliminate the vibration velocity and with substituting equation (3.3) in (3.2) yield

$$
\begin{gathered}
F=\Phi U+v Z_{\mathrm{m}}=\Phi U-F Z_{\mathrm{m}} / Z_{\mathrm{r}} \\
F\left(1+\frac{Z_{\mathrm{m}}}{Z_{\mathrm{r}}}\right)=U \Phi \\
F=U \Phi \frac{Z_{\mathrm{r}}}{Z_{\mathrm{r}}+Z_{\mathrm{m}}}=U \Phi \frac{Z_{\mathrm{R}} \cdot \Phi^{2}}{Z_{\mathrm{R}} \cdot \Phi^{2}+Z_{\mathrm{M}} \cdot \Phi^{2}}=U \Phi \frac{Z_{\mathrm{R}}}{Z_{\mathrm{R}}+Z_{\mathrm{M}}}
\end{gathered}
$$

On the other hand we have

$$
F=p A
$$

If we substitute (3.4) in (3.3) we have

$$
p A=U \Phi \frac{Z_{\mathrm{R}}}{Z_{\mathrm{R}}+Z_{\mathrm{M}}} /: U A
$$




$$
\frac{p}{U}=\frac{\Phi}{A} \frac{Z_{\mathrm{R}}}{Z_{\mathrm{R}}+Z_{\mathrm{M}}}
$$

and in accordance with the definition of transmitting voltage sensitivity $S(\mathrm{~Pa} / \mathrm{V} / / 1 \mathrm{~m})$ we have

$$
S=\frac{p}{U}=\frac{\Phi}{A} \frac{Z_{\mathrm{R}}}{Z_{\mathrm{R}}+Z_{\mathrm{M}}}
$$

So we have that the level of transmitting voltage sensitivity - LS is

$$
\begin{gathered}
L S=20 \log |S| \quad(\mathrm{dB} \text { re } 1 \mathrm{~Pa} / \mathrm{V} / / 1 \mathrm{~m}) \quad \text { or } \\
L S=20 \log |S|+120 \quad(\mathrm{~dB} \text { re } 1 \mu \mathrm{Pa} / \mathrm{V} / / 1 \mathrm{~m})
\end{gathered}
$$

By using (3.6) in the form of module value, we have that is

$$
L S=20 \cdot \log \left|\frac{\Phi}{A} \frac{Z_{R}}{Z_{R}+Z_{M}}\right|+120 \quad(\mathrm{~dB} \text { re } 1 \mu \mathrm{Pa} / \mathrm{V} / / 1 \mathrm{~m})
$$

Also, if we substitute the complex value of electric impedance $Z_{R}$ and $Z_{M}$ in equation (3.9) we get real and imaginary part of transmitting voltage sensitivity $S_{R e}$ and imaginary $S_{\operatorname{Im}}$ as follows

$$
\begin{gathered}
S_{\mathrm{Re}}=\frac{\Phi}{A} \frac{R_{\mathrm{R}}\left(R_{\mathrm{R}}+R_{\mathrm{M}}\right)}{\left(R_{\mathrm{R}}+R_{\mathrm{M}}\right)^{2}+x_{\mathrm{M}}{ }^{2}} \\
S_{\mathrm{Im}}=\frac{\Phi}{A} \frac{R_{\mathrm{R}} x_{\mathrm{M}}}{\left(R_{\mathrm{R}}+R_{\mathrm{M}}\right)^{2}+x_{\mathrm{M}}{ }^{2}}
\end{gathered}
$$

If the module of transmitting voltage sensitivity $|S|$ is given to (3.12)

$$
|S|=\sqrt{S_{\operatorname{Re}}^{2}+S_{\operatorname{Im}}^{2}}
$$

then in the end we have the level of transmitting voltage sensitivity as follows

$$
L S=20 \cdot \log |S|+120 \quad(\mathrm{~dB} \text { re } 1 \mu \mathrm{Pa} / \mathrm{V} / / 1 \mathrm{~m})
$$

where is

$S(\mathrm{~Pa} / \mathrm{V})$-transmitting voltage sensitivity $S=S_{\mathrm{Re}}+S_{\mathrm{Im}}$ of hydroacoustical transducer $|S|(\mathrm{Pa} / \mathrm{V})$-module of transmitting voltage sensitivity of hydroacoustical transducer $L S(\mathrm{~dB}$ re $1 \mu \mathrm{Pa} / \mathrm{V})$-level of transmitting voltage sensitivity of hydroacoustical transducer with references of pressure in water of $p_{0}=1 \mu \mathrm{Pa}$ and voltage of $1 \mathrm{~V}$, In accordance with equation (3.13) we can show given solves in a practical example as follows in Fig. 3.1. 


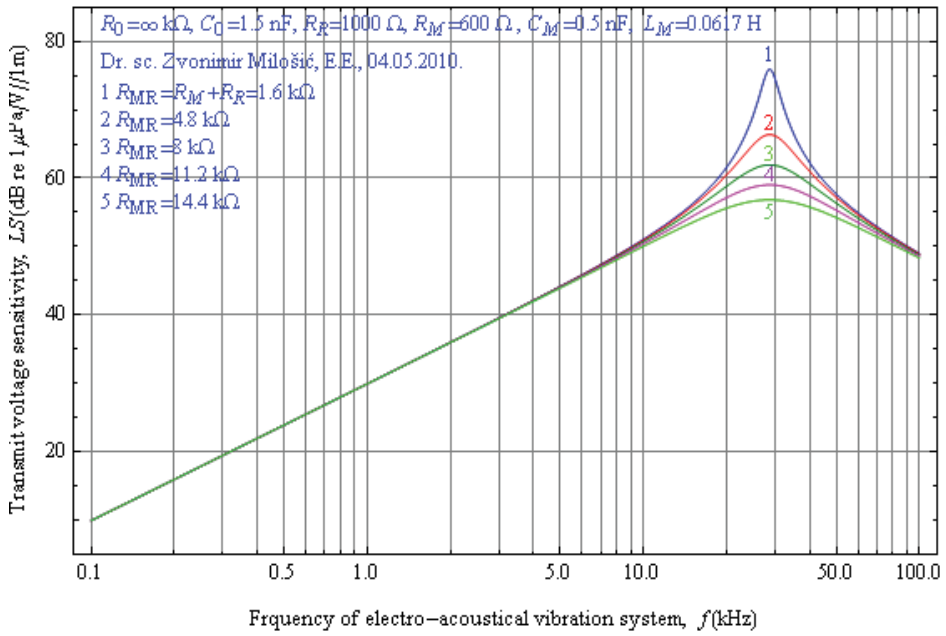

Fig. 3.1 Level of transmitting voltage sensitivity of piezoelectric transducer - LS

\section{The de-embedding of the level of the receive voltage sensitivity of transducers $-L M(\mathrm{~dB}$ re $1 \mathrm{~V} / \mu \mathrm{Pa})$}

By using equations (2.1.1), (2.1.2), (2.1.3) and (3.4) to Fig. 4.1 in the system with equivalent electric elements and $L C$ transformer we have that partial receive voltage sensitivity $M_{22}$ at port 2 and at clamps 2-2 is defined by real and imaginary parts of impedances as follows.

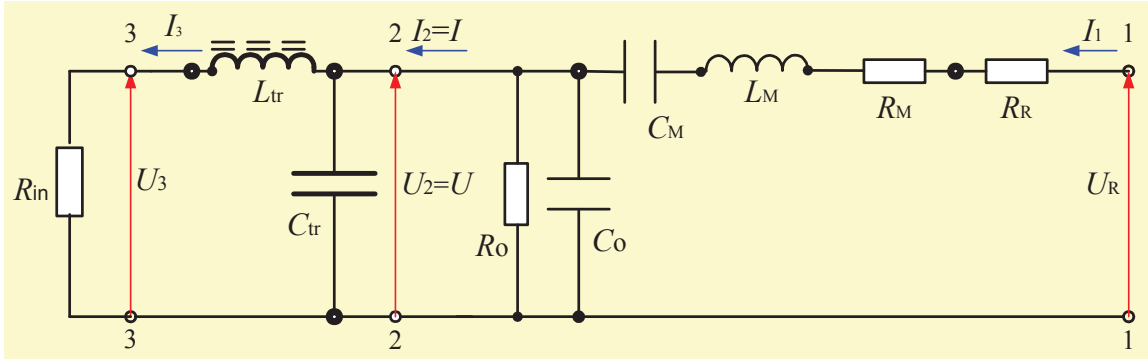

Fig. 4.1 Electric block scheme for general form of equation of electric parameters on input and electric analogies of mechanical parameters of vibration system with $L C$ transformer

$$
\begin{array}{ll}
M_{\mathrm{r} 22}=\frac{A}{\Phi} \frac{R_{0} X_{0}\left[R_{\mathrm{MR}} X_{0}+R_{0}\left(X_{0}+X_{\mathrm{M}}\right)\right]}{\left[R_{\mathrm{MR}} X_{0}+R_{0}\left(X_{0}+X_{\mathrm{M}}\right)\right]^{2}+\left(X_{0} X_{\mathrm{M}}-R_{\mathrm{MR}} R_{0}\right)^{2}} & (\mathrm{~V} / \mathrm{Pa}) \\
M_{\mathrm{i} 22}=\frac{A}{\Phi} \frac{R_{0} X_{0}\left(R_{0} X_{\mathrm{M}}-R_{\mathrm{MR}} X_{0}\right)}{\left[R_{\mathrm{MR}} X_{0}+R_{0}\left(X_{0}+X_{\mathrm{M}}\right)\right]^{2}+\left(X_{0} X_{\mathrm{M}}-R_{\mathrm{MR}} R_{0}\right)^{2}} & (\mathrm{~V} / \mathrm{Pa})
\end{array}
$$


These solves of receive voltage sensitivity have special solves with special values of resistor of losses $R_{0}$. If we divide (at real $M_{\mathrm{r} 22}$ and imaginary $M_{\mathrm{i} 22}$ ) the numerator and denominator with the product of $R_{0} X_{0}$ and for $R_{0}>>$, we can apply 'limes' with $R_{0} \rightarrow \infty$, yields

$$
\begin{aligned}
& \lim _{R_{0} \rightarrow \infty} M_{\mathrm{r} 22}=\frac{A}{\Phi} \cdot \frac{X_{0}\left(X_{0}+X_{\mathrm{M}}\right)}{R_{\mathrm{MR}}^{2}+\left(X_{0}+X_{\mathrm{M}}\right)^{2}} \quad(\mathrm{~V} / \mathrm{Pa}) \\
& \lim _{R_{0} \rightarrow \infty} M_{\mathrm{i} 22}=\frac{A}{\Phi} \cdot \frac{R_{\mathrm{MR}} X_{0}}{R_{\mathrm{MR}}^{2}+\left(X_{0}+X_{\mathrm{M}}\right)^{2}} \quad(\mathrm{~V} / \mathrm{Pa})
\end{aligned}
$$

By using real and imaginary part of receive voltage sensitivity in the de-embedding and plotting of characteristics of level of receive voltage sensitivity in the complex form of presentation, yields that the module of complex value is

$$
M_{22}=\sqrt{M_{\mathrm{r} 22}^{2}+M_{\mathrm{i} 22}^{2}} \quad(\mathrm{~V} / \mathrm{Pa})
$$

In accordance with the general definition for level of receive voltage sensitivity $L M_{22}$ at clamps $2-2$, at valid reference of $p_{0}=1 \mu \mathrm{Pa}$ in hydroacoustics, we have

$$
L M_{22}=20 \log M_{22}-120 \quad(\mathrm{~dB} \text { re } 1 \mathrm{~V} / \mu \mathrm{Pa})
$$

Also, in accordance with equation (4.6) we have graphic presentation for analysis of parameters and conditions for next steps. Fig. 4.2 shows us the functional dependence of the receive sensitivity of frequency with high impact of values of acoustic resistors in the character of form and of colored functions.

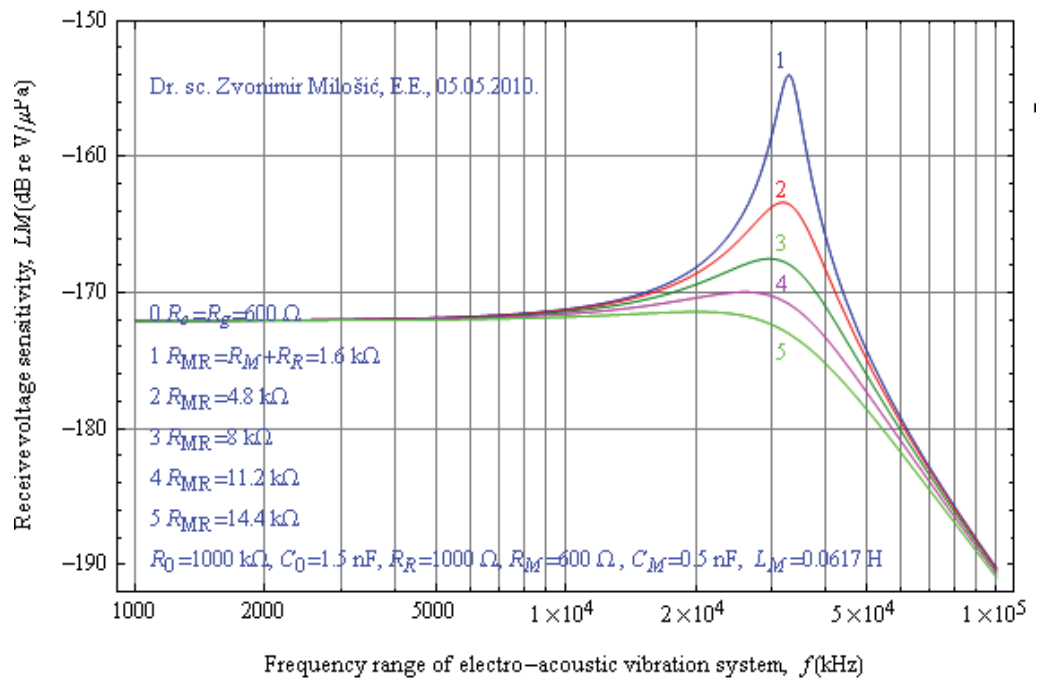

Fig. 4.2 Functional dependence of the level of the receive voltage sensitivity on frequency at different parameters contained in mechanical, electric and acoustical part of transducer body calculated with given numerical values inside figure 
In this part of the analysis we can also see the characteristic of the functional dependence of receive voltage sensitivity of different values of capacity at clamps 2-2 given in the form of $\mathrm{C}_{0}$ and shown in Fig. 4.3.

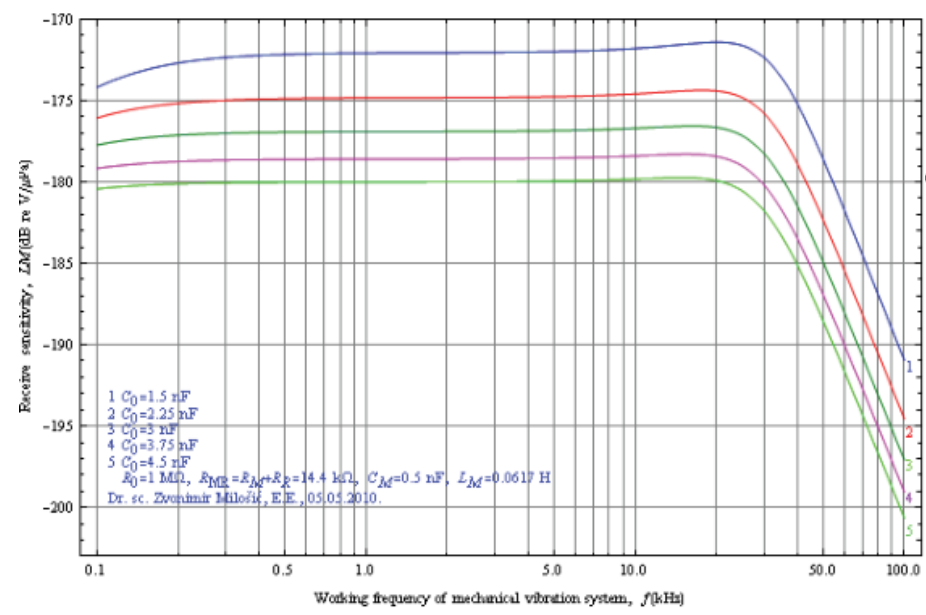

Fig. 4.3 Functional dependence of the level of the receive voltage sensitivity on the frequency at different parameters contained in mechanical, electric and acoustical part of transducer body calculated with given different numerical values of $C_{0}$ noticed inside figure

By using Fig. 4.1 we can write equations for the next step, the step of matching electric impedance of hydroacoustical transducer as a hydrophone. Then we have

$$
\begin{gathered}
I_{3}=\frac{U}{R_{\mathrm{c}}+\mathrm{j} X_{L \operatorname{tr}}} \\
U_{3}=I_{3} R_{\mathrm{c}} \\
U_{3}=U \frac{R_{\mathrm{c}}}{R_{\mathrm{c}}+\mathrm{j} X_{L \operatorname{tr}}} \\
U=U_{\mathrm{c}}\left(1+\mathrm{j} \frac{X_{L \operatorname{trr}}}{R_{\mathrm{c}}}\right) \\
U_{3}=U_{2} \frac{R_{\mathrm{c}}}{R_{\mathrm{c}}+\mathrm{j} X_{L \operatorname{tr}}}=U\left[\frac{R_{\mathrm{c}}{ }^{2}}{R_{\mathrm{c}}{ }^{2}+X_{L \operatorname{tr}}{ }^{2}}-\mathrm{j} \frac{R_{\mathrm{c}} X_{L \operatorname{lt}}}{R_{\mathrm{c}}{ }^{2}+X_{L \operatorname{tr}}{ }^{2}}\right] /: p \\
\frac{U_{3}}{p}=\frac{U}{p}\left[\frac{R_{\mathrm{c}}{ }^{2}}{R_{\mathrm{c}}{ }^{2}+X_{L t r}{ }^{2}}-\mathrm{j} \frac{R_{\mathrm{c}} X_{L \operatorname{lt}}}{R_{\mathrm{c}}{ }^{2}+X_{L t r}{ }^{2}}\right]
\end{gathered}
$$




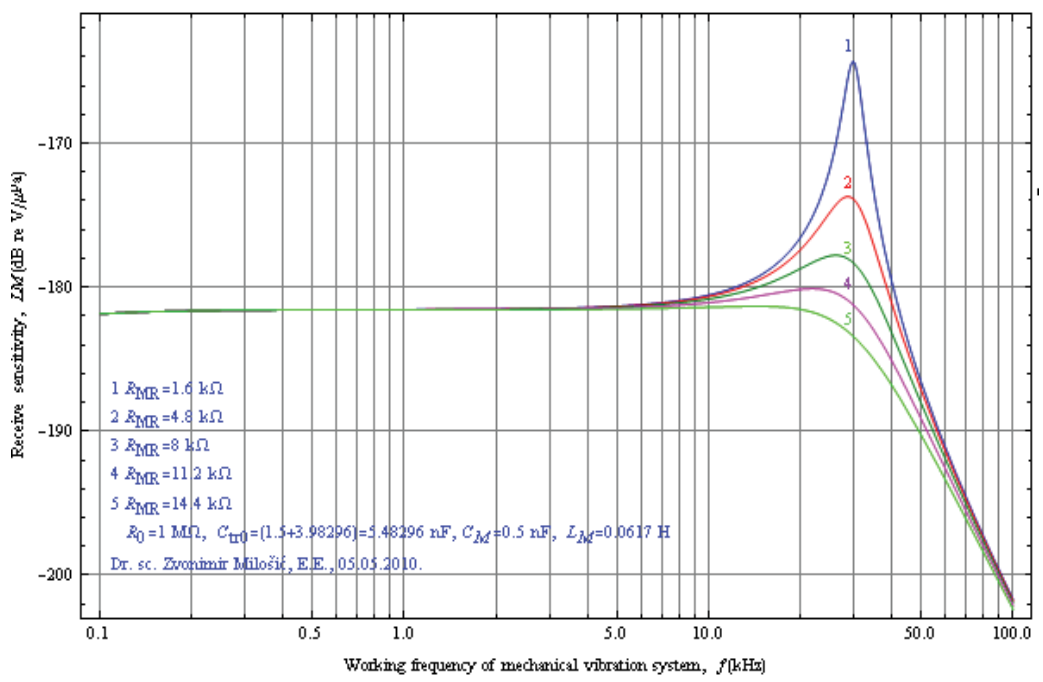

Fig. 4.4 Function of the level of the receive voltage sensitivity of hydrophone with $L C$ transformer of inner impedance $Z_{g}$ of hydrophone as a generator of electric signal

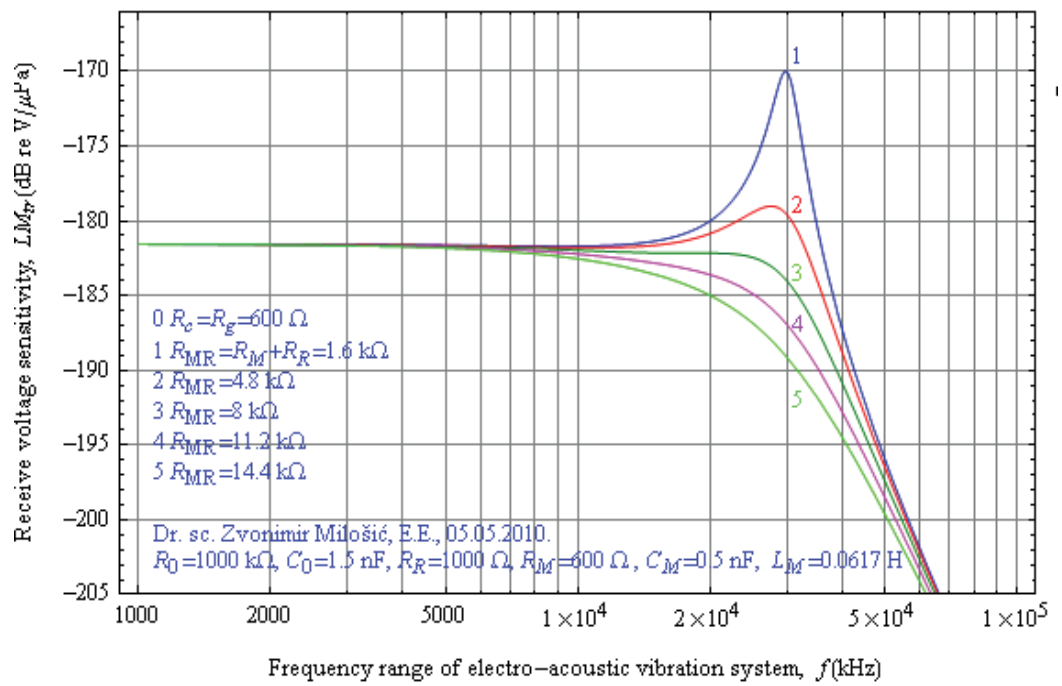

Fig. 4.5 Function of the level of the receive voltage sensitivity of hydrophone with $L C$ transformer of inner impedance depending on the frequency at different parameters contained in mechanical, electric and acoustic part of transducer body calculated with given different numerical values of resistance $R_{\mathrm{MR}}$ and resistance of electric cable $R_{\mathrm{c}}$ noticed inside figure 
At this moment we have two new receive voltage sensitivities: new virtual $M_{\mathrm{vir}}(\mathrm{V} / \mathrm{Pa})$ and new receive voltage sensitivity $M_{\mathrm{tr}}(\mathrm{V} / \mathrm{Pa})$ with $L C$ transformer. So, in the end, we must know that they are defined to (4.13) and (4.14)

$$
\begin{aligned}
& M_{\mathrm{tr}}=\frac{U_{3}}{p} \quad(\mathrm{~V} / \mathrm{Pa}) \\
& M_{\mathrm{vir}}=\frac{U}{p} \quad(\mathrm{~V} / \mathrm{Pa})
\end{aligned}
$$

Both of them can be calculated to given equations, which can also be measured in the hydroacoustical tank separately.

In accordance with the given equations we can calculate virtual receive voltage sensitivity to equation (4.5) with intervention in the new value of $X_{C_{\text {trCo }}}$ as a parallel connection of value $C_{\text {tr }}$ and $C_{0}$ or to equation (4.15) directly.

$$
\begin{gathered}
L M_{22 \text { vir }}=L M_{22}-20 \log \left(\frac{C_{\mathrm{tr}}+C_{0}}{C_{0}}\right)(\mathrm{dB} \text { re } 1 \mathrm{~V} / \mu \mathrm{Pa}) \\
M_{\mathrm{tr}}=M_{\mathrm{vir}}\left[\frac{R_{\mathrm{c}}{ }^{2}}{R_{\mathrm{c}}{ }^{2}+X_{L \mathrm{tr}}^{2}}-\mathrm{j} \frac{R_{\mathrm{c}} X_{L \mathrm{tr}}}{R_{\mathrm{c}}{ }^{2}+X_{L \mathrm{tr}}{ }^{2}}\right]=M_{\mathrm{vir}} \cdot(\alpha-\mathrm{j} \beta) \\
M_{\mathrm{tr}}=\left(M_{\mathrm{rvir}}+\mathrm{j} M_{\mathrm{ivir}}\right)(a-\mathrm{j} \beta)=\left(a M_{\mathrm{rvir}}+\beta M_{\mathrm{ivir}}\right)_{\text {retr }}+\mathrm{j}\left(a M_{\mathrm{ivir}}-\beta M_{\mathrm{rvir}}\right)_{\mathrm{imtr}}=M_{\text {retr }}+M_{\mathrm{imtr}}
\end{gathered}
$$

And now, the module of receive voltage sensitivity of hydroacoustical transducer is given to

$$
\left|M_{t r}\right|=\sqrt{M_{\text {retr }}^{2}+M_{\text {imtr }}^{2}} \quad(\mathrm{~V} / \mathrm{Pa})
$$

In the end, we have the level of voltage receive sensitivity of transducer, as follows

$$
L M_{\mathrm{tr}}=20 \log \left|M_{\mathrm{tr}}\right|-120 \quad(\mathrm{~dB} \text { re } 1 \mathrm{~V} / \mu \mathrm{Pa})
$$

It is good to say that in accordance with mentioned equations we will get a new function as follows in Fig. 4.5, where is voltage sensitivity approximately $11 \mathrm{~dB}$ less then at the beginning.

\section{The de-embedding of directivity pattern characteristic $R_{p}(\lambda, \theta, \varphi$, LoSoS) of antenna}

In accordance with the previous matter, we also have directivity pattern as one of important parameters of acoustical transducers. Directivity pattern of one hydroacoustical transducer, and directivity pattern in array of antenna are fundamental roots of all analyses in the systems where they are installed. The de-embedding of all parameters of systems begins with main parameters of hydroacoustical directivity pattern characteristic of composite transducers separately or transducers in antenna array.

By using this model of coordinate system to show and define the beginning conditions for de-embedding or the measurement of different functions of directivity pattern 
characteristics of antennas at frequency from infrasound to ultrasound range, we can make correct analyses of all parameters in the system without restricted parameters of producers and data of technology owner.

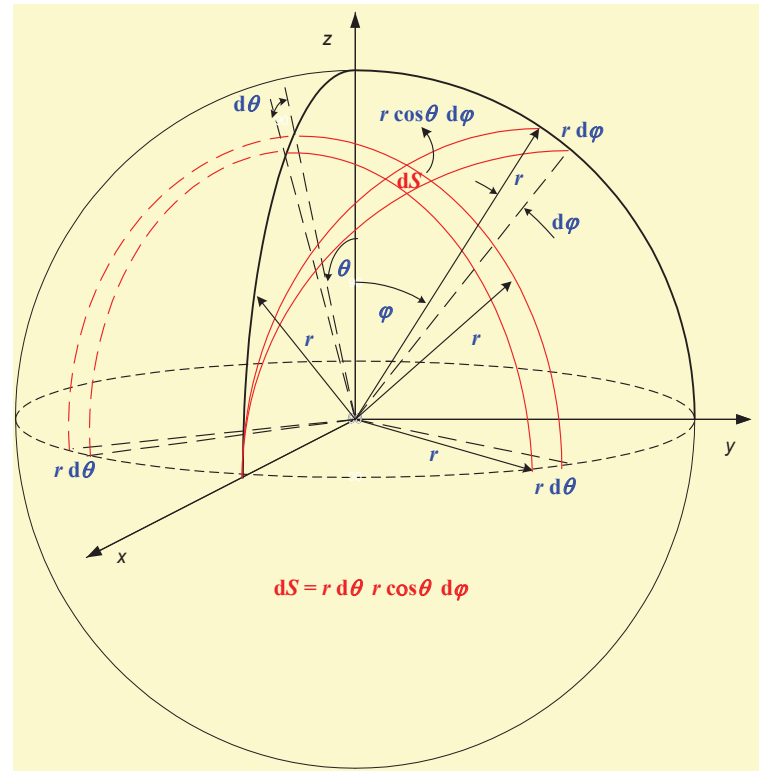

Fig. 5.1 Coordinate system to show and define the beginning conditions in all derivations and analyses of contemporary systems of monitoring with special review on underwater technology, $\mathrm{d} S=r^{2} \cos \theta \mathrm{d} \theta \mathrm{d} \varphi$

Keeping in line with this intention, we can talk about a composite cylindrical barrel antenna, mainly installed in the bulb at bow of great tankers for monitoring of possibly forthcoming dangerous rocks, crags and shallows in the sea, or not less simple composed antenna array of ultrasound scanners in medicine. It is contained in equations taken over from literature (Evtjutov \& Mitko 1981, Sverdlin 1980, Milošić 1996) in short explanation as follows

By using equation (5.1) (Evtjutov \& Mitko 1981) we can get directivity pattern characteristics for different model of contemporary sonar horn antenna as follows

$$
R p=20 \log \left\{\frac{D_{2}^{2}}{\left(D_{2}^{2}-D_{1}^{2}\right)} \cdot\left\{\frac{2 J_{1}\left(\frac{\Pi \cdot D_{2}}{\lambda} \cdot \sin \theta\right)}{\frac{\Pi \cdot D_{2}}{\lambda} \cdot \sin \theta}-\left(\frac{D_{1}}{D_{2}}\right)^{2} \cdot \frac{2 J_{1}\left(\frac{\Pi \cdot D_{1}}{\lambda} \cdot \sin \theta\right)}{\frac{\Pi \cdot D_{1}}{\lambda} \cdot \sin \theta}\right\}\right\}
$$



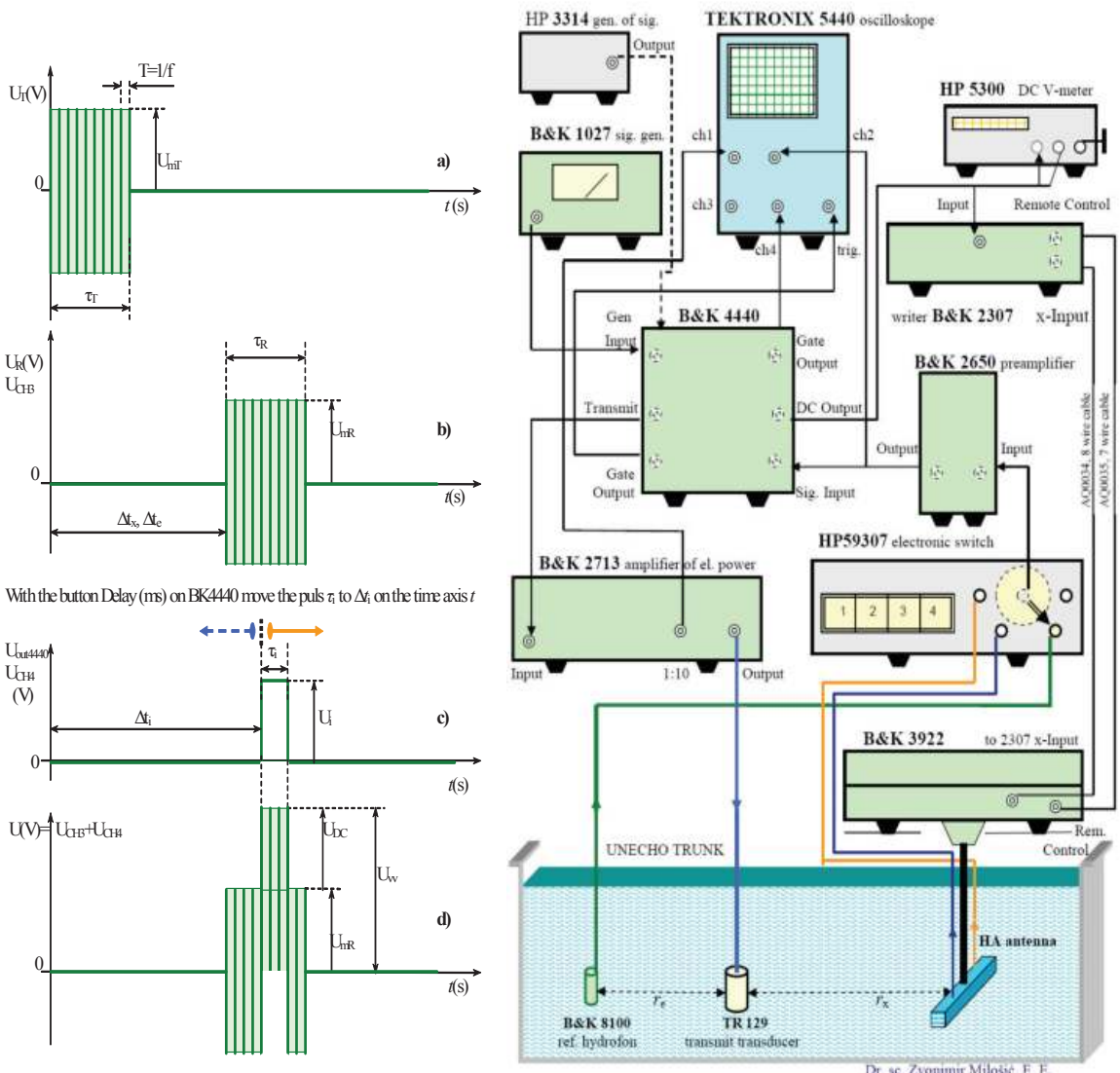

With the button Delay $(\mathrm{ms})$ on BK4440 move the puls $\tau_{\mathrm{i}}$ to $\Delta_{\mathrm{i}}$ on the time axis $t$
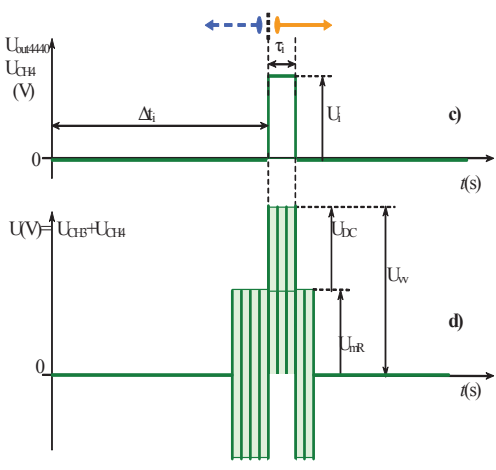

Dr. sc. Zvonimir Milokic, E. E.

Fig. 5.2 Model of block scheme of impulse method for measurement of receive voltage sensitivity characteristics and directivity pattern characteristics of antenna showing electric pulses in the real time domain in the process of calibration (BI, Zagreb)
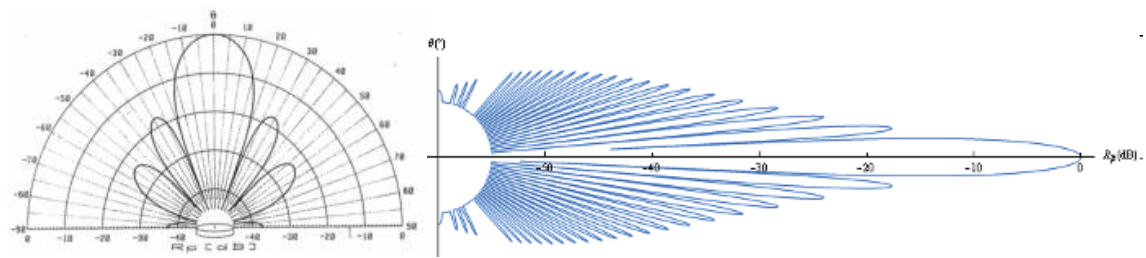

Fig. 5.3 Two different directivity pattern characteristics of baffled circle piston (Milošić 1988/2010 to equations of Evtjutov\&Mitko 1981, Smarišev 1973, Sverdlin 1976) 


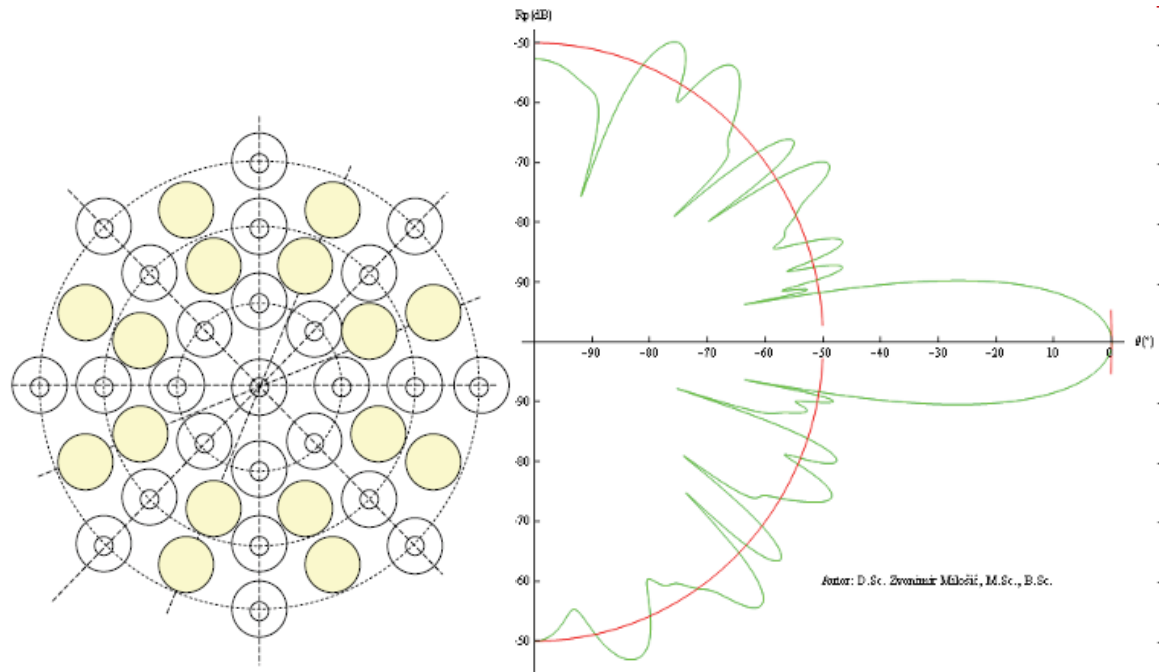

Fig. 5.4 Physical contain of forty transducers in specific form of circle series in symmetric positions with belonging function of directivity pattern characteristic of antenna (Milošić 2008)

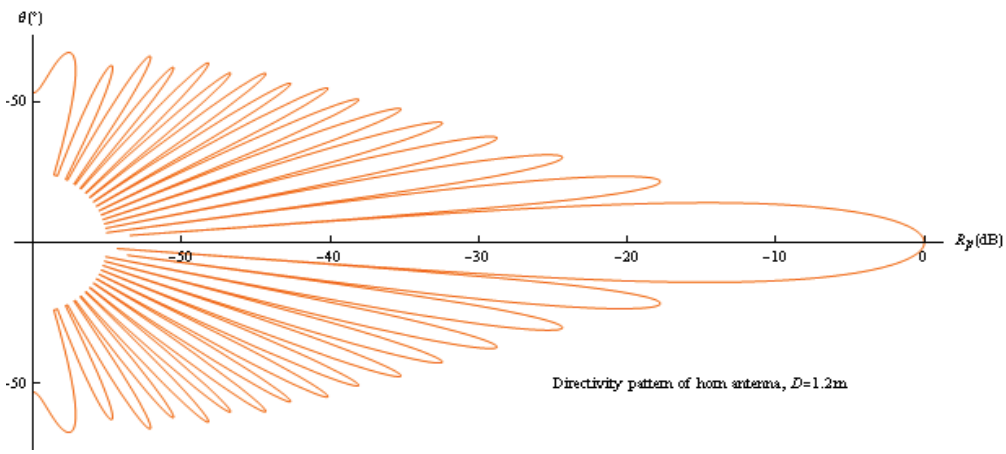

Fig. 5.5 Directivity pattern characteristic of contemporary horn antenna at relations of outside diameter and wave length $D_{2} / \lambda=16$ to (5.1)

\section{The new impact of directivity pattern characteristic $R_{p}(\lambda, \theta, \varphi$, LoSoS) on directivity index and effective aperture $A_{\text {eff }}(\lambda, \theta, \varphi$, LoSoS), of antenna}

Depending on a model of antenna (linear array, circle array, barrel array or spherical array), of working frequency $f(\mathrm{~Hz})$, of their transmitting power $P(\mathrm{~W})$, of efficiency of antenna in receive or in transmitting $\eta(1)$, to general known definition, we can have three forms of directivity index in receive $D I_{R}(\mathrm{~dB})$ or in transmit $D I_{\mathrm{T}}(\mathrm{dB})$ and effective aperture of antenna in receive $A_{\text {effR }}$ or in transmit $A_{\text {effT }}$ as follows: at rectangle cross section - $\mathrm{CS}_{\mathrm{r}}$ of main beam of 
pattern, at circle cross section - $\mathrm{CS}_{\mathrm{c}}$ and at elliptical cross section of main lobe of pattern characteristic - $\mathrm{CS}_{\mathrm{e}}$. In accordance with three models of cross sections of main lobe of pattern characteristic of antenna, mentioned above, we have five different new equations for Directivity Index-DI and five new functions for Effective Aperture- $A_{\text {eff }}$ in antenna theory (Milošić 2004, 2005, 2007 and 2008). On the other hand, we have graphical presentation in two and three dimensions of both mentioned parameters for different conclusions, for practice. In accordance with equation $(6.1)$ at the beginning, we have fundamental definition (Urick 1967, 1975, 1998, Smarišev 1973, Sverdlin 1976, Burdic 1984,)1 as the most suitable at new equations (Milošić 2004, AAAA2005, Acoustics'08 Paris) for rectangle, circle and ellipse CS (cross section) of main lobe with parameter LoSoS (Level of Suppression of Side lobes as negative value) in all equations to Fig. 5.1 and to 'general definitions'2, as follows

$$
\begin{gathered}
A G=D I=10 \cdot \log \frac{\int_{4 \Pi} \mathrm{d} \Omega}{\int_{4 \Pi} R_{I}(\theta, \varphi) \mathrm{d} \Omega}=10 \cdot \log \frac{4 \Pi}{\int_{0}^{2 \pi} \int_{-\Pi / 2}^{+\Pi / 2} R_{I}(\theta, \varphi) \cdot \cos \theta \cdot \mathrm{d} \theta \cdot \mathrm{d} \varphi} \\
D I=10 \cdot \log D_{\mathrm{f}}(\lambda, \theta, \varphi, L o S o S)(\mathrm{dB}) \\
A_{\text {eff }}=\frac{\lambda^{2}}{4 \Pi} D_{\mathrm{f}}(\lambda, \theta, \varphi, L o S o S)\left(\mathrm{m}^{2}\right)
\end{gathered}
$$

Also, we have equations for effective aperture $A_{\text {eff }}$ as new function with parameter $L o S o S$.

\subsection{New function for directivity index $D I_{\mathrm{r}-\mathrm{cs}}$ on the basis of theory at rectangle cross section $\mathrm{CS}_{\mathrm{r}}$ of main lobe of receive or transmit directivity pattern characteristic}

In accordance with the mentioned condition in Fig. 5.1 we have correct values of DI at idealized directivity pattern characteristics in Fig. 6.1.1.

Equation (6.1.1) is absolutely correct at given condition of rectangle CS of main lattice

$$
D I_{\text {rectangle_CS }}=10 \log \frac{2 \Pi}{\left(1-10^{\frac{\text { LoSoS }}{10}}\right) \cdot \varphi_{-3 \mathrm{~dB}} \sin \left(\frac{\theta_{-3 \mathrm{~dB}}}{2}\right)+2 \Pi \cdot 10^{\frac{\text { LoSoS }}{10}}}(\mathrm{~dB}) \text { (Milošić 2004) }
$$

\footnotetext{
${ }^{1}$ Before about 1948, directivity index was regarded as negative instead of a positive quantity (Urick R. J., Peninsula Publishing, California, Los Altos, $3^{\text {rd }}$ edition, page 42).

${ }^{2}$ By using the mathematical coordinate system (by all authors), at $\mathrm{d} S=r^{2} \sin \theta \mathrm{d} \theta \mathrm{d} \varphi$ as differential surface, they had a big problem to make a join of suppression of side lobes and directivity index in one function at contemporary sonar systems. It is important to point out Russian scientist Grigorii M. Sverdlin as one of the first authors who introduced so called 'rectangle approximation' in directivity pattern characteristic in Fig. 3.28 in the de-embedding of directivity index, at wrong equation 3.76, published in the book from 1976.
} 

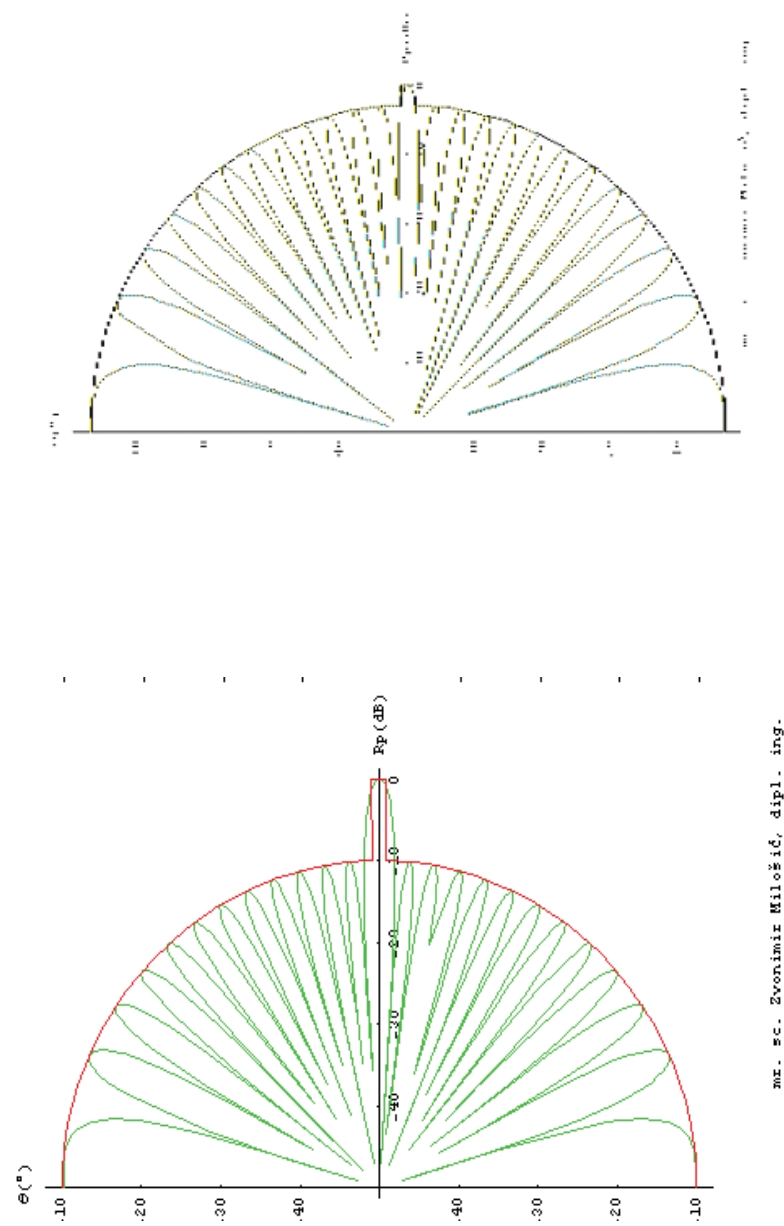

Fig. 6.1.1 Directivity patterns of the theoretical sonar antenna at azimuth angle $\theta_{-3 \mathrm{~dB}}=1^{\circ}$ and elevation angle $\varphi-3 \mathrm{~dB}=15^{\circ}$ and with $L o S o S=-3 \mathrm{~dB}$ we have $D I=3 \mathrm{~dB}$, and also in the second example in azimuth of $\theta_{-3 \mathrm{~dB}}=1^{\circ}$ and elevation angle $\varphi_{-3 \mathrm{~dB}}=15^{\circ}$ and with $L o S o S=-10 \mathrm{~dB}$ and in that case we have that is $D I=10 \mathrm{~dB}$ 

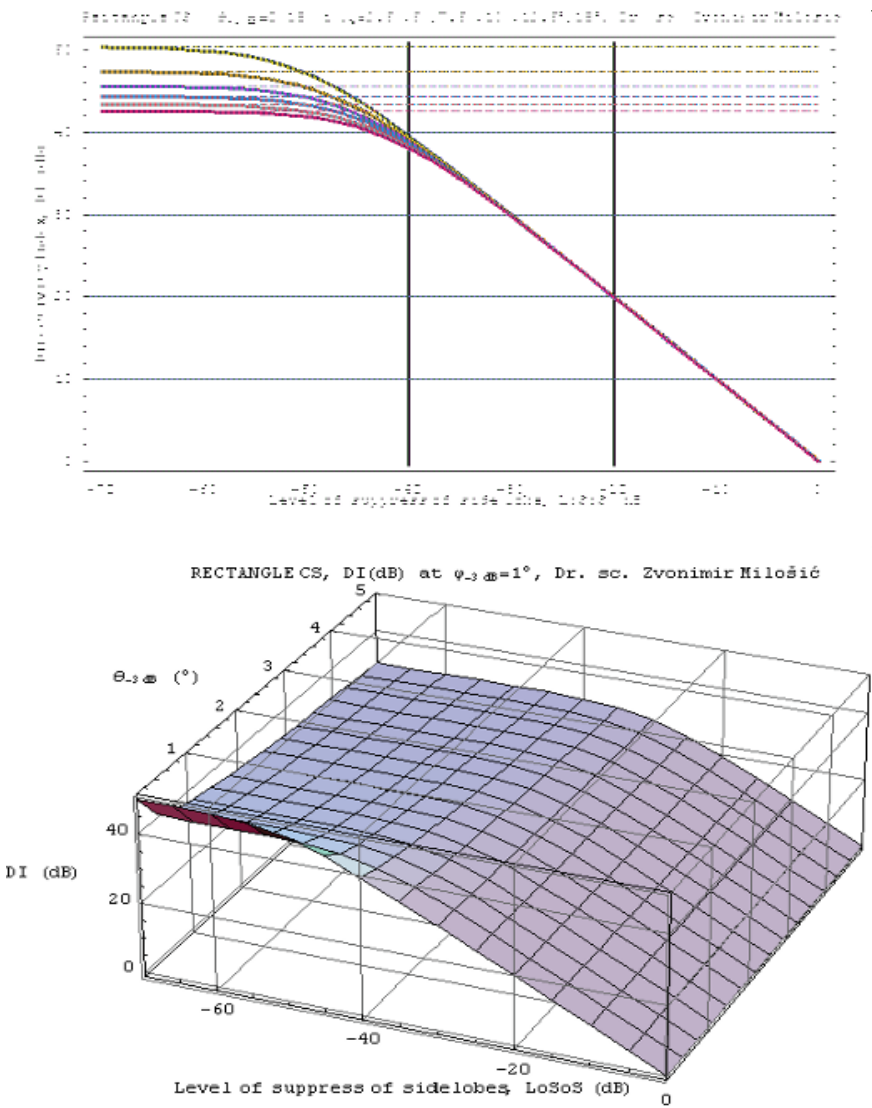

Fig. 6.1.2 Directivity index shown in two and three dimensions to (6.1.1) as correct function for theory and practice in dependence of LoSoS (dB) (Milošić 2004, 2005, 2007)

Directivity index of ideal mathematical case to (6.1.2) is mainly in use in theory and in practice as a beginning point in analysis. But, this value is mainly one of the largest wishes, which produces approximate error of directivity index to $20 \mathrm{~dB}$. Taking the product of ideal directivity factor $D_{\mathrm{f}}(1)$ and efficiency factor $\eta(1)$ of antenna as final value of array gain or directivity index is additionally wrong. Efficiency factor and directivity factor are absolutely different parameters in the root (Caruthers page 27). Absolutely all solves of directivity index, effective aperture or array gain of antenna are contained in equations (6.1.1), (6.2.1) and (6.3.1) or in contemporary figures Fig. 6.1.2 to Fig. 6.3.5, (Milošić 2004, 2005, 2007, 2008).

$$
D I_{\text {rectangla_CS }}=10 \cdot \log D_{\mathrm{f}}=10 \cdot \log \frac{41253}{\varphi_{-3 \mathrm{~dB}}\left({ }^{\circ}\right) \theta_{-3 \mathrm{~dB}}\left({ }^{\circ}\right)}(\mathrm{dB})
$$


6.2 New function for directivity index $D I_{c-c s}$ on the basis of theory at circle cross section $\mathrm{CS}_{\mathrm{c}}$ of main lobe of receive or transmit directivity pattern characteristic

Both equations, (6.2.1) when used in practice and (6.2.2) as mathematical ideal function are new in theory and practice for circle CS of main lattice given and explained in the paper at Acoustics'08 (Milošić 2008).
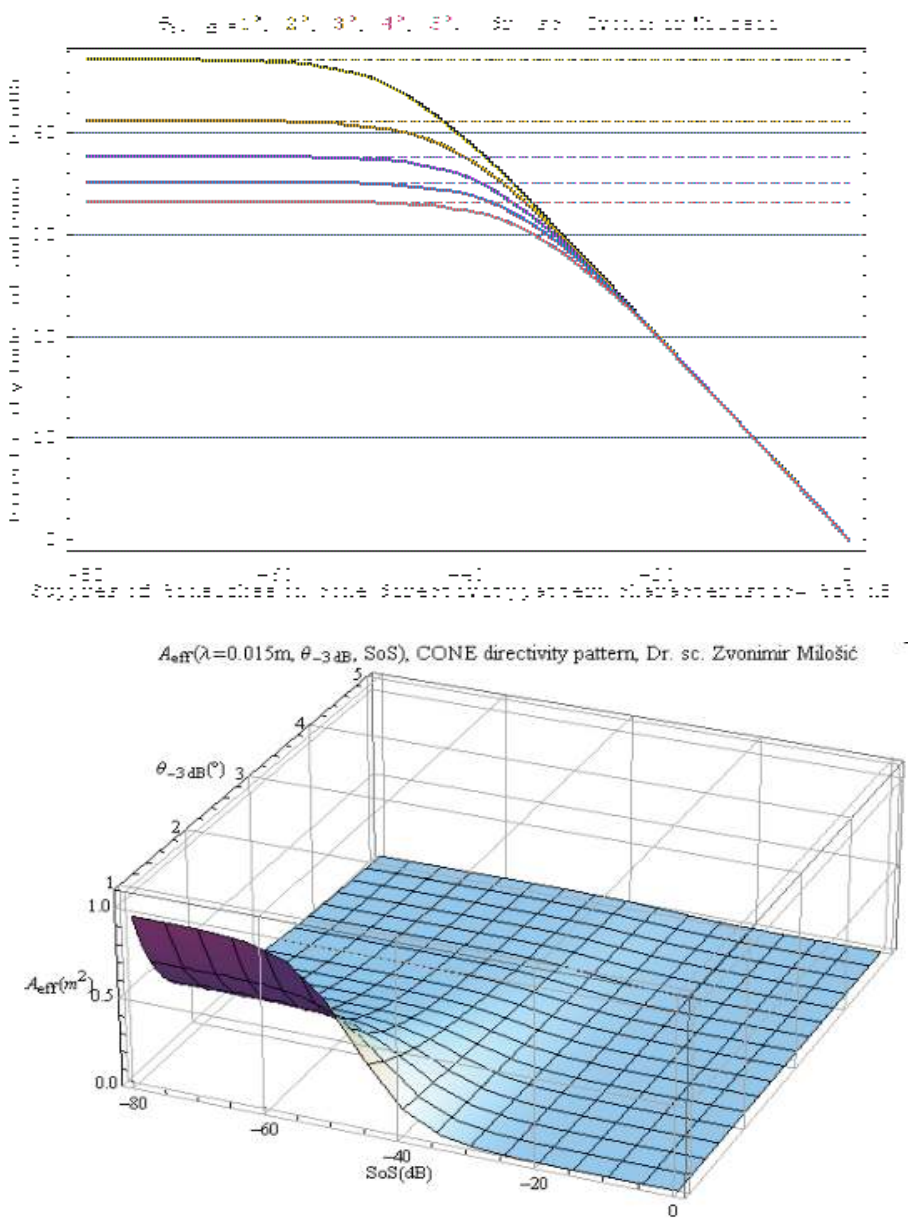

Fig. 6.2.1 Directivity index and effective aperture of antenna at cone directivity pattern characteristic in condition of circle CS of main lobe (Milošić 2008)

$$
D I_{\text {circle_CS }}=10 \log \frac{1}{\left(1-10^{\frac{\text { LoSoS }}{10}}\right) \cdot \sin ^{2}\left(\frac{\theta_{-3 \mathrm{~dB}}}{4}\right)+10^{\frac{\text { LoSoS }}{10}}}
$$

(dB) (Milošić Acoustics'08) (6.2.1) 


$$
\left.D I_{\text {circle_CS }}=10 \cdot \log \frac{52525}{\theta_{-3 \mathrm{~dB}}^{2}\left({ }^{\circ}\right)}(\mathrm{dB}) \text { (Milošić 2008, Acoustics' } 08\right)
$$

In accordance with mentioned equations (6.2.1) and (6.2.2) for circle CS of the main lobe of pattern characteristic of antenna, we have graphic presentation in Fig. 6.2.1 for DI in two dimensions and for $A_{\text {eff }}$ in three dimensions.

\subsection{New function for directivity index $D l_{\text {e-cs }}$ on the basis of theory at ellipse cross section $\mathrm{CS}_{\mathrm{c}}$ of main lobe of receive or transmit directivity pattern characteristic}

In absolute accordance with the mentioned conditions of rectangle and circle cross section in this case, we have the best general function for the presentation of important parameters of antennas in the systems of monitoring, in the underwater or in the air.

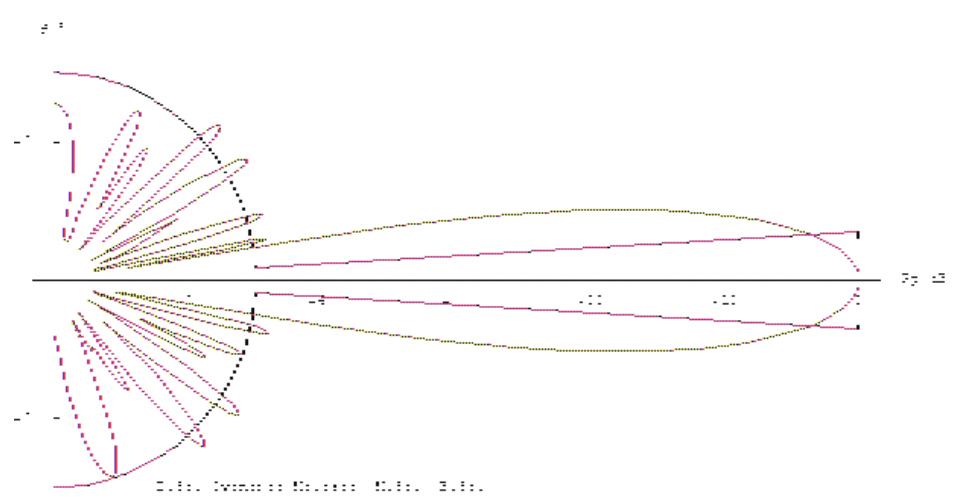

Fig. 6.3.1 Directivity patterns of sonar antenna at elliptic CS of the main lobe, if we have azimuth angle $\theta_{-3 \mathrm{~dB}}=4.8^{\circ}$ and the value of given elevation angle $\varphi_{-3 \mathrm{~dB}}=15^{\circ}$ then with the level of suppression of minor lobes of characteristic $L o S o S=-45 \mathrm{~dB}$ we have that is $D I=28.5 \mathrm{~dB}$

$$
\begin{aligned}
D I_{\text {ellipse_CS }}= & 10 \cdot \log \frac{8}{\left(1-10^{\frac{\text { LoSoS }}{10}}\right) \cdot \varphi_{-3 \mathrm{~dB}} \sin \left(\frac{\theta_{-3 \mathrm{~dB}}}{2}\right)+8 \cdot 10^{\frac{\text { LoSoS }}{10}}}(\mathrm{~dB})(\text { Milošić 2007)(6.3.1) } \\
& D I_{\text {ellipse_CS }}=10 \cdot \log \frac{52525}{\varphi_{-3 \mathrm{~dB}}\left({ }^{\circ}\right) \theta_{-3 \mathrm{~dB}}\left({ }^{\circ}\right)} \quad(\mathrm{dB})(\text { Milošić 2007) }
\end{aligned}
$$

That is a general equation (6.3.1) (Milošić Workbench 2007 in Germany published \& recessional unrealized OCEANS'09 \& recessional unrealized in 158 ${ }^{\text {th }}$ ASA meeting 2009 \& recessional unrealized Euronoise09 \& recessional unrealized at ECUA2010) for using in theory and practice at all conditions of environmental influence and new equation (6.3.2), mainly as very high and unattainable value. 


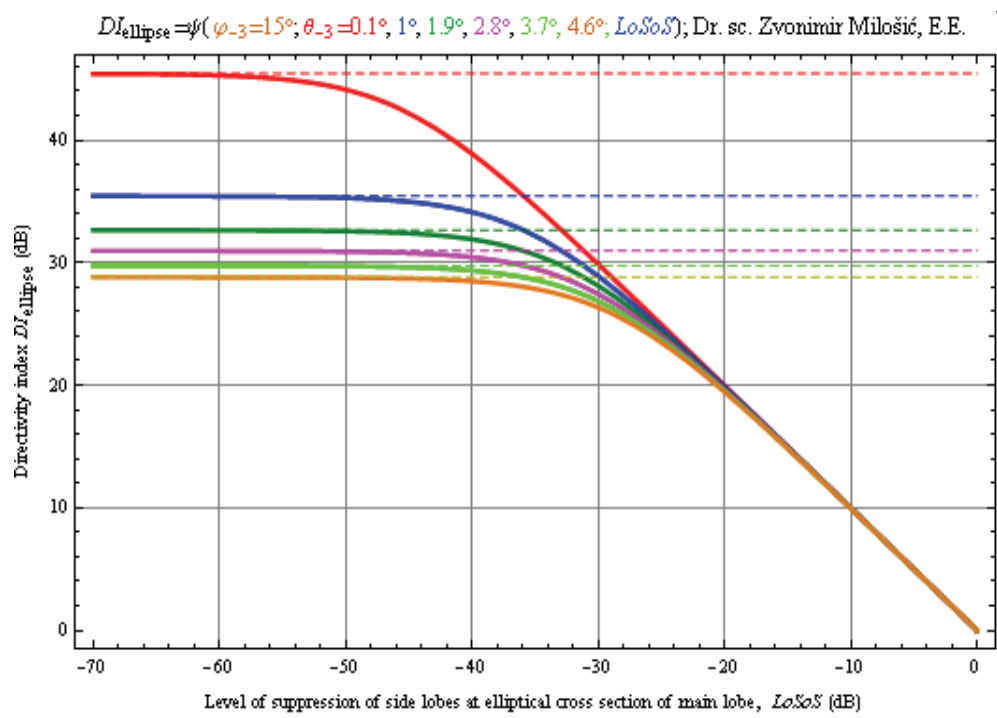

Fig. 6.3.2 Graphic presentation of $D I$ as function of angles of sensitivity $\theta_{-3 \mathrm{~dB}}$ in azimuth and $\varphi$-3dв in elevation and the level of suppression of side lobes LoSoS of the sonar antenna (Milošić 2007, 2008)

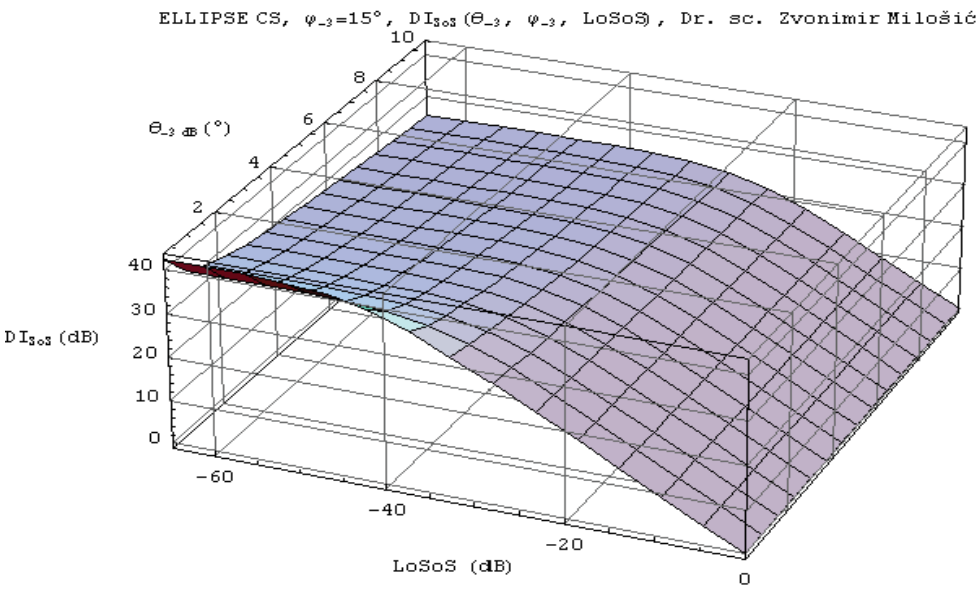

Fig. 6.3.3 Graphic presentation of $D I\left(\theta_{-3 \mathrm{~dB}}, \varphi_{-3 \mathrm{~dB}}, L o S o S\right)$ in three dimensions have a special meaning by comparison of different sonar systems (Milošić 2007, 2008) 


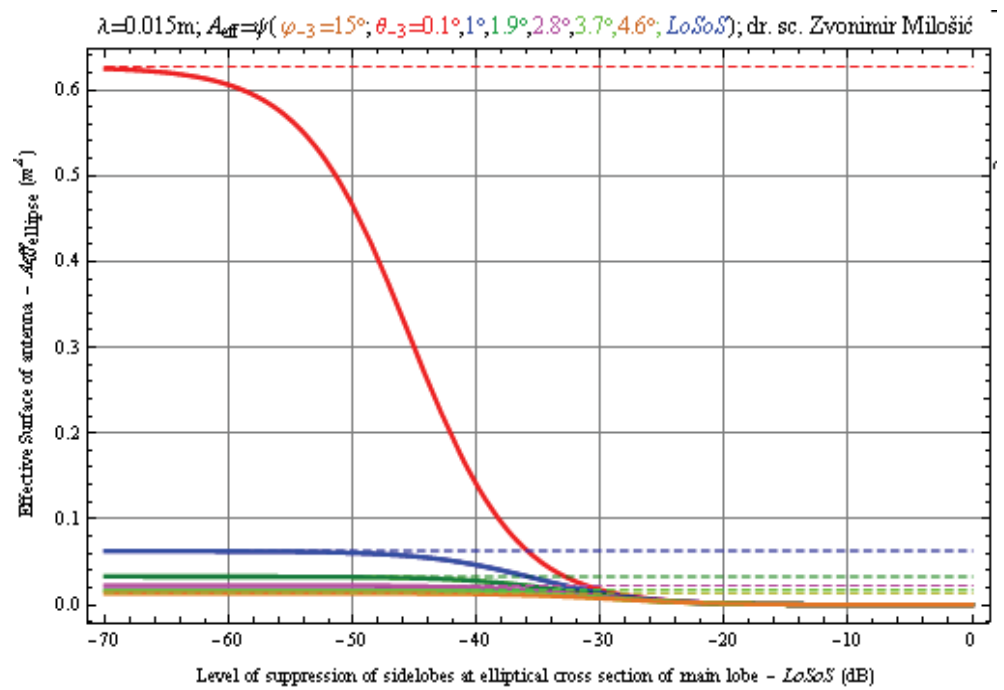

Fig. 6.3.4 Graphic presentation of an effective aperture of antenna $A_{\text {eff }}\left(\theta_{-3 \mathrm{~dB}}, \varphi_{-3 \mathrm{~dB}}, L_{0} S_{o} S\right)$ in two dimensions at the model with elliptical cross section (CS) (Milošić 2007, 2008)

ELLIPSE CS, $\lambda=15 \mathrm{~mm}, \varphi_{-3}=15^{\circ}, A_{\mathrm{eff}}\left(\lambda, \theta_{-3}, \varphi_{-3}\right.$, LoSoS), Dr. sc. Zvonimir Milošić

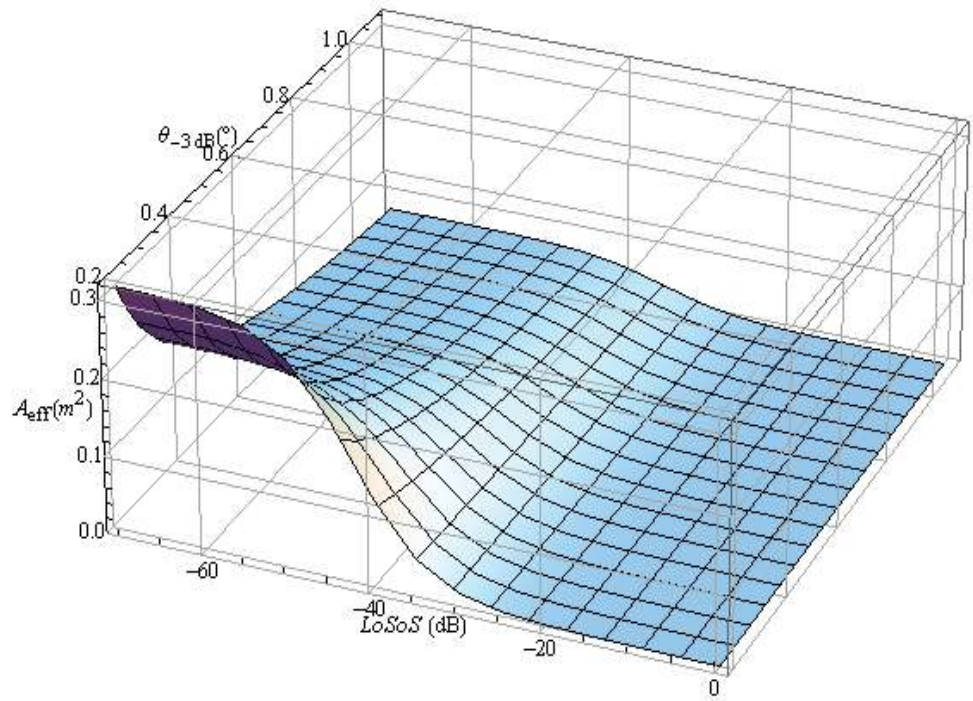

Fig. 6.3.5 Graphic presentation of an effective aperture of antenna $A_{\mathrm{eff}}\left(\lambda, \theta_{-3 \mathrm{~dB}}, \varphi_{-3 \mathrm{~dB}}, L_{0} S o S\right)$ in three dimensions at the model with elliptical cross section (Milošić 2007, 2008) 


\section{Conclusion}

The presented model of vibration of medium with concentrated mechanical parameters as a starting point of analysis of transmitting or receive sensitivity of hydroacoustical transducer with mathematical solution of its differential equation is applicably carried out on the basis of the Laplace transformation of complex variable. Given analyses of antenna with concentrated mechanical parameters are in accordance with mathematical solutions by twoport network model of hydroacoustical transducer with original concentrated electric parameters and with the electric substitute of mechanical parameters inside the body, at electrical clamp on one side and on the surface of the transducer in the water on the second acoustical side.

With the analysis of the behaviour of mathematical functions of hydroacoustical sensitivity and sources of wide dispersion of phase angle of impedance of antenna elements at hydroacoustical and ultrasound transducers, we can choose the best model for optimal matching in the given sonar system. By using the ideal electric transformer models, special, original and general expressions were gained and tested in the long-time practice, we have enabled the correct computing of elements necessary for performance and the complete matching of electrical impedance at hydroacoustical antenna. In accordance with the mentioned intentions of presenting fundamental sensitivities of transducers, electroacoustical impedance and their matching, we have presented a new important expanded and correct opinion about the fundamentals of directivity index and effective aperture of antenna in a new form of absolutely correct mathematical functions dependent on important parameter LoSoS. Also, all of them in two and three dimensional graphs are the best support in the building of antennas and new technologies of contemporary systems of monitoring for a world without accidents in space and, especially, in the underwater.

\section{References}

Aronov L. M., Matveev L. I. (1953); Fundaments of hydroacoustic, Navy Edition, Moscow, in Russian \& in Croatian,

Beranek, L. Leo (1954); ACOUSTICS, MIT, Bolt, Beranek and Newman, Inc., Cambridge, Massachusetts, New York Toronto London, USA,

Bobber J. Robert (1998); Underwater Electroacoustic Measurements, Peninsula Publishing, Los Altos, California, USA,

Bogorodskii V.V, Zubarev L. A., Korepin E. A., Jakušev V. I.(1983); Underwater electroacoustic transducers, Sudostroenie, in Russian,

Bronstein I. N., Semendjaev K. A. (1975); Mathematical handbook for engineers and students, translate from Russian Danilo Blanuša, Tehnička knjiga, Zagreb, in Croatian,

Brüel \& Kjær (1980); Introduction to underwater acoustics, hydrophones - their characteristics and applications calibration technique, Copenhagen, Denmark,

Burdic S. William (1984); Underwater acoustic system analyses, Prentice-Hall, Inc., Englewood Cliffs, New Jersey, USA,

Caruthers W. Jerald (1977); Fundamentals of marine acoustics, Elsevier Scientific Publishing Company, Amsterdam-Oxford-New York,

Evtjutov P. Alesandr, Mitko B. Valerii (1981, 1988); Examples of engineer computing in hydroacoustics, Sudostroenie, in Russian, 
Jelaković, Tihomil (1967); Introduction to the basics electrical engineering, Tehnička knjiga Zagreb, in Croatian,

Jelaković, Tihomil (1973); Transistors audio amplifiers, Školska knjiga Zagreb, in Croatian,

Kinsler E. L., Frey R. A. (1962); Fundamentals of acoustics, John Wiley\&Sons, Inc., USA,

Kolesnikov Aleksei Evgenievič (1983); Acoustical measurements, Sudostroenie, in Russian,

Loeser, T. Harrison (1998); Sonar engineering handbook, Peninsula Publishing, Los Altos, California,

Masson P. Waren (1950); Piezoelectric cristals and their applications to ultrasonics, D. Van Nostrand Co., New York, USA,

Milošić, Zvonimir (1978); Matching electrical impedance of hydroacoustic transducers at a stable working frequency, Elektrotehnika, XXII-1978, No. 3-6, pages 171-173, in Croatian,

Milošić, Zvonimir (1988); Analysis of negative impact roll and pitch of ship on the measurement of its velocity with ultrasound Doppler log, ETAN 1988 International Congress in Zadar,

Milošić Zvonimir (1990); Physics of Ultrasound and diagnostical instrumentation in hepatogastroenterology, Chapter I, pp. 9-45, Edited by Živković R. \& Bilić A., Application of ultrasound in clinical hepatology and gastroenterology, Školska knjiga Zagreb, in Croatian,

Milošić Zvonimir (1993); Comments on 'Diffraction constants of acoustic transducers', J. Acoust. Soc. Am. 36, 267-269 (1964), J. Acoust. Soc. Am., Vol. 93, No. 2,

Milošić, Zvonimir (1996); Directivity pattern analysis of the ultrasound cone antenna by means of the model of a rigid hole circular piston in a rigid baffle, 38th International Symposium, Electronics in Marine, Elmar June 1996, Zadar, CROATIA,

Milošić, Zvonimir (2004); Models for parameter de-embedding of hydroacoustic antennas in underwater monitoring systems, Dissertation, FER Zagreb, in Croatian,

Milošić, Zvonimir (2005); Directivity index of a hydroacoustical antenna with an idealized model of a directivity pattern, 2nd Congress of Alps-Adria Acoustics Association and 1st Congress of Acoustical Society of Croatia, 23-24 June 2005, Opatija, Proceedings AAAA 2005, ISBN 953-95097-0-X, see ending of Proceedings Acoustics'08 Paris,

Milošić, Zvonimir (2007); The Importance of the influence of directivity index of antenna on the range at multistatic sonar systems in conditions of reverberation in the sea and with the use on radar systems, Workbench in FGAN, pp 1-35, Germany,

Milosić, Zvonimir (2008); "Suppression of side lobe level on the cone characteristics of the directivity pattern of an antenna as an important factor in its directivity index and effective aperture", 1. Proceedings of the 9th ECUA, Volume 1\&2, pp. 533-538, 2.Proceedings of 2nd ASA-EAA joined conference integrated with Societe Francaise d'Acoustique 2008, on DVD pages 4821-4826, Editor Manell E. Zakharia and co-editors: Didier Cassereau and Francine Luppe (ur.), Acoustics'08 Paris, France,

Milošić, Zvonimir (2010); Ideal electrical transformer model in the system of impedance matching of piezoelectric hydroacoustic transducer, Journal of Marine Sciences, Edited by University of Dubrovnik, Naše more 57(1-2) 2010,

http:/ / www.nasemore.com/sadrzaj/brojevi/57(1-2)2010/4-milosic.pdf

Rževkin, Sergei Nikolaevič (1960); Course of lessons in theory of sound, Edited by Moscow University, in Russian,

Scherman H. Charls, Butler L.John (2008); Transducers and Arrays for Underwater Sound, sponsored by ONR, published by Springer, 
Smarišev, Mihail Dmitrievič (1973); Hydroacoustic antenna directivity pattern, Edited by Sudostroenie, Leningrad, in Russian,

Sverdlin, M. Grigorii (1976); Applied hydroacoustic, Sudostroenie, Leningrad, in Russian,

Sverdlin Mihailovič Grigorii (1980); Hydroacoustic transducers and antennas, Sudostroenie, Leningrad, in Russian,

Urick J. Robert (1975); Principles of underwater sound, McGraw-Hill, Book Company 1975, Science redactor E. L. Šenderov, Redactor Committee: Kolesnikov-ProstakovSmarišev-Tarasjuk, translated in Russian,

Urick J. Robert (1998); Principles of underwater sound, 3rd edition, Peninsula Publishing, Los Altos, California 1998, USA,

Wilson, B. Oscar (1998); Introduction to the theory and design of sonar transducers, Peninsula Publishing, Los Altos, California, USA,

Wolfram Research; Europe Ltd., Mathematica 4 \& 6, 10 Blenheim Office Park, Lover Road, Long Hanbourgh, Oxfordshire OX29 8RY, UK.; SYSTEMCOM d.o.o., certified reseller for Wolfram Research, Maksimirska 120, 10000 Zagreb, CROATIA, for Microsoft Windows 95/98/NT/ 2000/XP,

Ziomek J. Lawrence (1995); Fundamentals of acoustic field theory and space-time signal processing, Naval Postgraduate School, Monteray, California, 1995 CRC Press Inc., USA. 
(c) 2010 The Author(s). Licensee IntechOpen. This chapter is distributed under the terms of the Creative Commons AttributionNonCommercial-ShareAlike-3.0 License, which permits use, distribution and reproduction for non-commercial purposes, provided the original is properly cited and derivative works building on this content are distributed under the same license. 